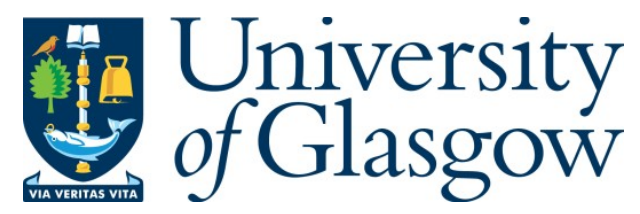

Hashlamoun, K., Abusara, Z., Ramirez Torres, A. , Grillo, A., Herzog, W. and Federico, S. (2020) Fluorescence recovery after photobleaching: direct measurement of diffusion anisotropy. Biomechanics and Modeling in Mechanobiology, 19(6), pp. 2397-2412.

(doi: 10.1007/s10237-020-01346-z)

There may be differences between this version and the published version. You are advised to consult the publisher's version if you wish to cite from it.

https://eprints.gla.ac.uk/238159/

Deposited on: 7 April 2021

Enlighten - Research publications by members of the University of Glasgow http://eprints.gla.ac.uk 


\title{
Fluorescence Recovery After Photobleaching: Direct Measurement of Diffusion Anisotropy
}

\author{
Kotaybah Hashlamoun ${ }^{1,2,3}$, Ziad Abusara ${ }^{2,4}$, Ariel Ramírez-Torres ${ }^{5}$, Alfio \\ Grillo $^{5}$, Walter Herzog ${ }^{1,2}$, and Salvatore Federico ${ }^{1,2}$ \\ ${ }^{1}$ Department of Mechanical and Manufacturing Engineering, The University \\ of Calgary, Calgary, Canada \\ ${ }^{2}$ Human Performance Laboratory, The University of Calgary, Calgary, Canada \\ ${ }^{3}$ Graduate Programme in Biomedical Engineering, The University of Calgary, \\ Calgary, Canada \\ ${ }^{4}$ (Present address) Advanced Imaging and Histopathology Core, Qatar \\ Biomedical Research Institute, Hamad Bin Khalifa University, Qatar \\ Foundation, P.O. Box 34110 Doha, Qatar \\ ${ }^{5}$ Dipartimento di Scienze Matematiche "G. L. Lagrange" Politecnico di \\ Torino, 10129. Torino, Italia
}

\begin{abstract}
Fluorescence recovery after photobleaching (FRAP) is a widely used technique for studying diffusion in biological tissues. Most of the existing approaches for the analysis of FRAP experiments assume isotropic diffusion, while only a few account for anisotropic diffusion. In fibrous tissues, such as articular cartilage, tendons and ligaments, diffusion, the main mechanism for molecular transport, is anisotropic, and depends on the fibre alignment. In this work, we solve the general diffusion equation governing a FRAP test, assuming an anisotropic diffusivity tensor and using a general initial condition for the case of an elliptical (thereby including the case of a circular) bleaching profile. We introduce a closed-form solution in the spatial coordinates, which can be applied directly to FRAP tests to extract the diffusivity tensor. We validate the approach by measuring the diffusivity tensor of $3 \mathrm{kDa}$ FITC-dextran in porcine medial collateral ligaments. The measured diffusion anisotropy was $1.42 \pm 0.015(\mathrm{SE})$, which is in agreement with that reported in the literature. The limitations of the approach, such as the size of the bleached region and the intensity of the bleaching, are studied using COMSOL simulations.
\end{abstract}

Keywords FRAP anisotropic diffusion fibrous tissues ligaments direct measure

\section{Introduction}

Molecular diffusion is the process by which chemical species, e.g., solutes or macromolecules, move from regions of higher concentration to regions of lower concentration. Diffusion plays a vital role in cellular functions, such as protein-protein interactions and metabolism [18]. In porous connective tissues such as ligaments and cartilage, diffusion is one of the primary mechanisms for nutrient transport. For this reason it has been extensively studied in healthy and degraded tissues $[14,3,20,19,10,11]$. 
Several techniques can be used for measuring self or molecular diffusivity (or diffusion coefficient in the isotropic case) of solutes in biological tissues: fluorescence correlation spectroscopy (c.f., [12]), single-particle tracking (c.f., [15]), and diffusion tensor MRI (c.f., $[9,13])$ ). However, the most common method is Fluorescence Recovery After Photobleaching (FRAP), as it requires less instrumentation than the other approaches (e.g., confocal microscopes), and diffusivity can be directly quantified.

In general, in a FRAP experiment, a tissue is stained with fluorescently labelled molecules, and a region of interest (ROI) is photobleached using a high intensity laser beam causing irreversible photochemical bleaching of the ROI. As a result, the fluorescence intensity detected by the microscope drops in the ROI. Due to the Brownian motion, the surrounding labelled molecules will eventually be transported into the ROI, restoring the intensity. By analysing the fluorescence recovery pattern over time, a direct measurement of diffusivity is obtained [1].

The first mathematical model describing FRAP was developed by [1] and has been adopted in the literature with some variations. The model, which is derived for isotropic diffusion in a plane, considers the bleaching over a circular spot defined in an $(x y)$-plane, and occurs uniformly throughout the $z$-axis, and thus, the recovery is two-dimensional. Besides the theory, the model by [1] provided a guideline to evaluate diffusivity from experiments, where diffusivity is obtained as a function of the dimension of the ROI (radius, if it is a circle) and the characteristic time of recovery. Early models generally assumed isotropic diffusion. With the widespread use of confocal microscopy, and its application in FRAP experiments, the analysis was adapted from the conventional FRAP to confocal FRAP $[7,8]$, but assumed isotropic diffusion. [11] proposed a technique that detects diffusion anisotropy in collageneous tissues, termed Continuous Point Bleaching. In this technique, a single pixel is bleached for a prolonged period of time, and the resulting bleached region, approximated by an ellipse, gives a direct measure of the diffusion anisotropy. This method can be used to find diffusion anisotropy without the diffusion magnitude. Based on spatial Fourier analysis, [16] proposed a model that can capture diffusion anisotropy in two-dimensional confocal FRAP experiments. The analysis is performed in the wave-vector domain (sometimes loosely called the "frequency domain", in analogy with the case of time-frequency analysis). The intensity, and therefore the concentration, is normalised with respect to the intensity of the bleached image in its initial condition. The normalised function is then the fundamental solution of the diffusion equation. The fitting is performed in the wave-vector domain to obtain the diffusivity tensor.

In this work, we propose to introduce a direct approach that can be applied in the spatial coordinates to detect and evaluate diffusion anisotropy. The method is based on solving the diffusion equation for the case of anisotropic diffusion, using an appropriate initial condition, and allowing for data fitting in the spatial coordinates. Moreover, we show that the moment of inertia tensor of the concentration can be seen as a promising quantity for detecting and measuring anisotropy.

To test the validity of the approach, we performed a set of FRAP experiments on medial collateral ligament (MCL) explants collected from porcine knee joints. Testing was performed on medial collateral ligaments since they have strong fibre alignment (see Figure 1), which leads to anisotropic diffusivity. Since our model describes transport by passive diffusion, we tested dextran molecules as they are inert and uncharged. However, the diffusivity might still be influenced by interactions with the matrix, e.g., binding, and also by the cells [10]. As for the size of the test molecule, we used $3 \mathrm{kDa}$ dextran (with a hydrodynamic radius of $1.4 \mathrm{~nm}$, Sigma-Alrdich) as it could be viewed as a molecule of intermediate size compared to the small therapeutics used for osteoarthritis, such as NSAIDs and steroids (with hydrodynamic radii smaller than $0.55 \mathrm{~nm}$ ), and the relatively large therapeutics, such as growth factors and antibodies (up to $5 \mathrm{~nm}$ in hydrodynamic radius, see [5] for more details). The choice of both ligaments and $3 \mathrm{kDa}$ dextran provides a basis for comparison of the results with existing literature [11]

Additionally, we performed COMSOL sensitivity simulations by varying the size of the bleached region, and the intensity of bleaching. The fitting procedure, which was performed over an idealised unbounded domain, implies an error, evaluated by numerically simulating the diffusion over a realistically bounded domain, in order to address the accuracy of the analytical method. 

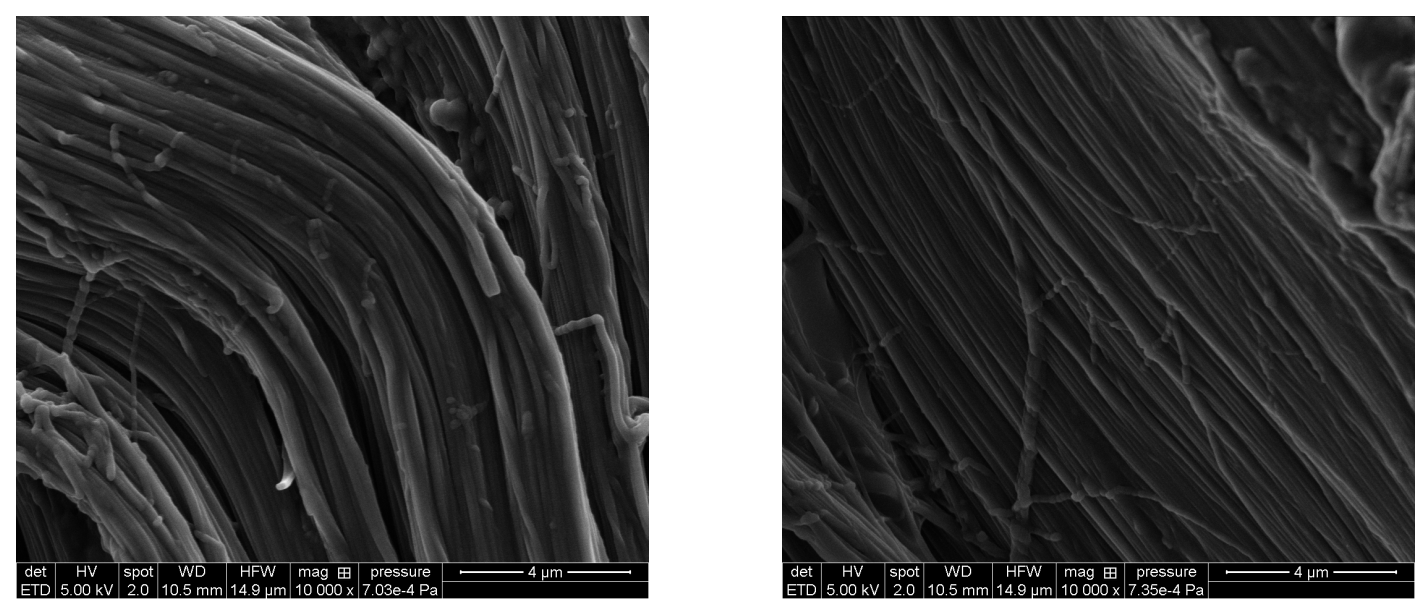

Figure 1: Scanning electron microscope (SEM) images of a medial collateral ligament sample, showing the alignment of the fibre bundles in two different locations. Note the $4 \mu \mathrm{m}$ scale bar at the bottom right of the image.

\section{Fluorescence Recovery After Photobleaching}

Here, we briefly recall the basics on Fluorescence Recovery After Photobleaching (FRAP). FRAP consists of staining the sample of interest with fluorescently-labelled molecules (or with fluorophores). It is usually assumed that the molecule of interest is large enough compared with the conjugated fluorescent probes, so that the diffusion observed is representative of the molecule of interest. A FRAP test occurs in three steps, explained here for a confocal microscope, but the same steps also apply to any light microscope $[1,17]$ :

1. Prebleaching: imaging of the sample at low power laser intensity. The laser is set at the wavelength at which fluorescent probes are excited. We will refer to these images as prebleached images/frames (Figure 2, left);

2. Bleaching: a region of interest (ROI), usually of circular shape, is bleached with a high power laser beam over a short period of time, so that recovery is minimal during the bleaching process. This yields a photochemical reaction that permanently causes the probes in the ROI to be nonfluorescent. We will refer to this image as bleached image/frame (Figure 2, middle);

3. Postbleaching: as a result of Brownian motion, the molecules carrying intact fluorophores are transported from the region surrounding the bleached ROI into the ROI, and the molecules inside the ROI move into the surrounding region and, thus, the fluorescence intensity inside the ROI is restored. We will refer to these images as postbleached images/frames (Figure 2, right).

In Figure 3, a schematic diagram summarises the steps that will be followed in this work: tissue preparation, testing, fitting the experimentally determined concentration, analytical/numerical determination of the diffusivity.

\section{Methods}

In this section, we determine the analytical expression of the concentration of the molecules during the recovery phase of a FRAP experiment for the case of anisotropic diffusion. We assume that the initial distribution is Gaussian, and consider both a circular and an elliptical ROI.

\subsection{Analytical Expression of the Recovery Profile}

The recovery observed in FRAP experiments is due to molecular diffusion, which can be described in terms of the mass balance law

$$
\partial_{t} c=\operatorname{div}(\boldsymbol{D} \operatorname{grad} c),
$$



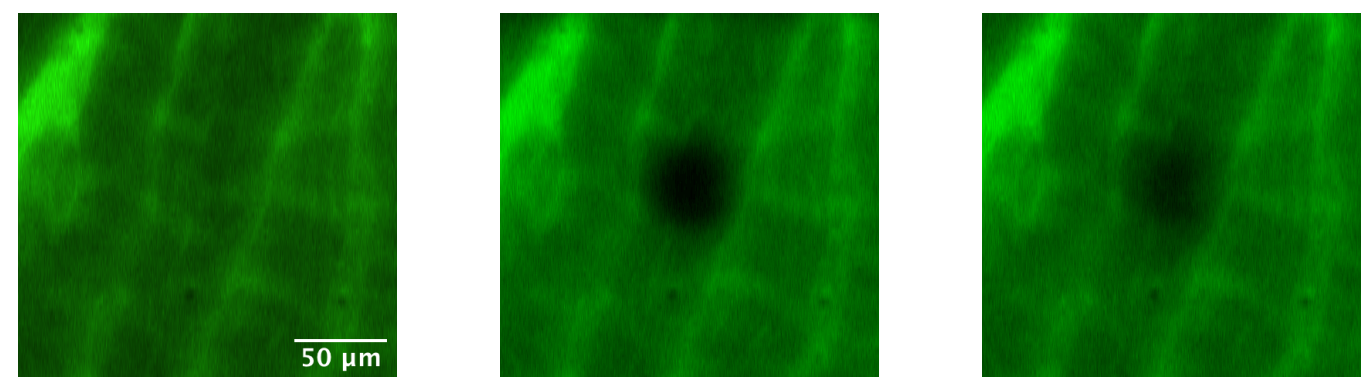

Figure 2: Steps of fluorescence recovery after photobleaching (FRAP) showing the prebleached image (left, $t<0$ ), the bleached image (middle, $t=0$ ), and a postbleached image (right, $t>0$; in this specific image, $t \simeq 1.5 \mathrm{~s}$ ). The images were taken for a medial collateral ligament sample, and acquired with a $40 \times / 1.1 \mathrm{NA}$ objective mounted on a Zeiss 710 inverted microscope (Zeiss Inc., Germany).

where $c$ is the concentration of the molecules, $\boldsymbol{D}$ is the diffusivity tensor and Fick's law is adopted to express the molecules' diffusive mass flux vector as $-\boldsymbol{D} \operatorname{grad} c$. Note that, in the present context, Equation (1) is assumed to apply to the whole two-dimensional Euclidean space $\mathbb{E}^{2}$. In our problem, the concentration is prescribed to tend towards a constant value $c_{\infty}$ (referring to the "unbleached condition") far away from the bleached region, i.e.,

$$
\lim _{\left\|\boldsymbol{x}-\boldsymbol{x}_{0}\right\| \rightarrow \infty} c(\boldsymbol{x}, t)=c_{\infty},
$$

and its gradient, $\operatorname{grad} c$, is required to have vanishing magnitude in the same limit, i.e.,

$$
\lim _{\left\|\boldsymbol{x}-\boldsymbol{x}_{0}\right\| \rightarrow \infty}\|\operatorname{grad} c(\boldsymbol{x}, t)\|=0 .
$$

We notice that in (2) and (3) the limits are assumed to exist independent of the direction along which $\boldsymbol{x}$ moves away from the centre of the bleached region, $\boldsymbol{x}_{0}$.

The initial concentration $c(\boldsymbol{x}, 0)$, occurring at the instant of bleaching, $t=0$, and resulting from a confocal laser, is empirically approximated by

$$
\begin{aligned}
c(\boldsymbol{x}, 0) & \equiv c_{0}(\boldsymbol{x}) \\
& =c_{\infty}\left[1-k \exp \left(-2\left(\boldsymbol{x}-\boldsymbol{x}_{0}\right) \cdot \boldsymbol{A}^{-1}\left(\boldsymbol{x}-\boldsymbol{x}_{0}\right)\right)\right],
\end{aligned}
$$

for all $\boldsymbol{x}, \boldsymbol{x}_{0} \in \mathbb{E}^{2}$, where $k$ is the bleaching depth parameter [1, 8] and $\boldsymbol{A}$ is a symmetric and positive definite second-order tensor. The bleached region is denoted by

$$
\mathcal{B}:=\left\{\boldsymbol{x} \in \mathbb{E}^{2}:\left(\boldsymbol{x}-\boldsymbol{x}_{0}\right) \cdot \boldsymbol{A}^{-1}\left(\boldsymbol{x}-\boldsymbol{x}_{0}\right) \leq 1\right\},
$$

i.e., it is the region bounded by the curve $\partial \mathcal{B}$, given by the ellipse of equation $\left(\boldsymbol{x}-\boldsymbol{x}_{0}\right) \cdot \boldsymbol{A}^{-1}\left(\boldsymbol{x}-\boldsymbol{x}_{0}\right)=1$.

Remark 1. Ideally, when considering a circular ROI, a perfectly isotropic Gaussian distribution of intensity (and thus, concentration) is to be adopted as the initial condition, in which case the tensor $\boldsymbol{A}$ of Equation (4) is isotropic, and is thus represented by the matrix

$$
[A]=\left[\begin{array}{cc}
r^{2} & 0 \\
0 & r^{2}
\end{array}\right],
$$

where $r$ is the effective radius of the circular bleached area, which is generally greater than the set bleaching radius [8]. Equation (6) allows us to re-write Equation (4) as

$$
c(\boldsymbol{x}, 0)=c_{\infty}\left[1-k \exp \left(-2 \frac{\left\|\boldsymbol{x}-\boldsymbol{x}_{0}\right\|^{2}}{r^{2}}\right)\right],
$$

which is the form usually found in the literature [7]. However, in order to account for possible anisotropy during the bleaching process, we consider a generally anisotropic tensor $\boldsymbol{A}$ in the initial 


\section{Tissue preparation}
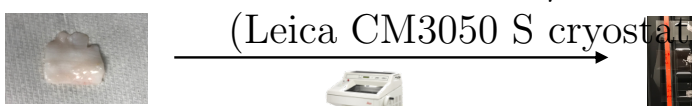

1. Healthy porcine
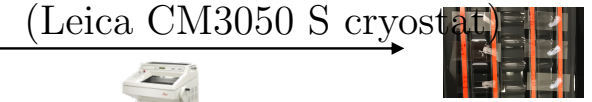

(Olympus FVMPE-RS)

medial collateral ligament

\section{$\longrightarrow$}

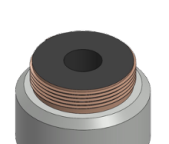

II. Testing - FRAP

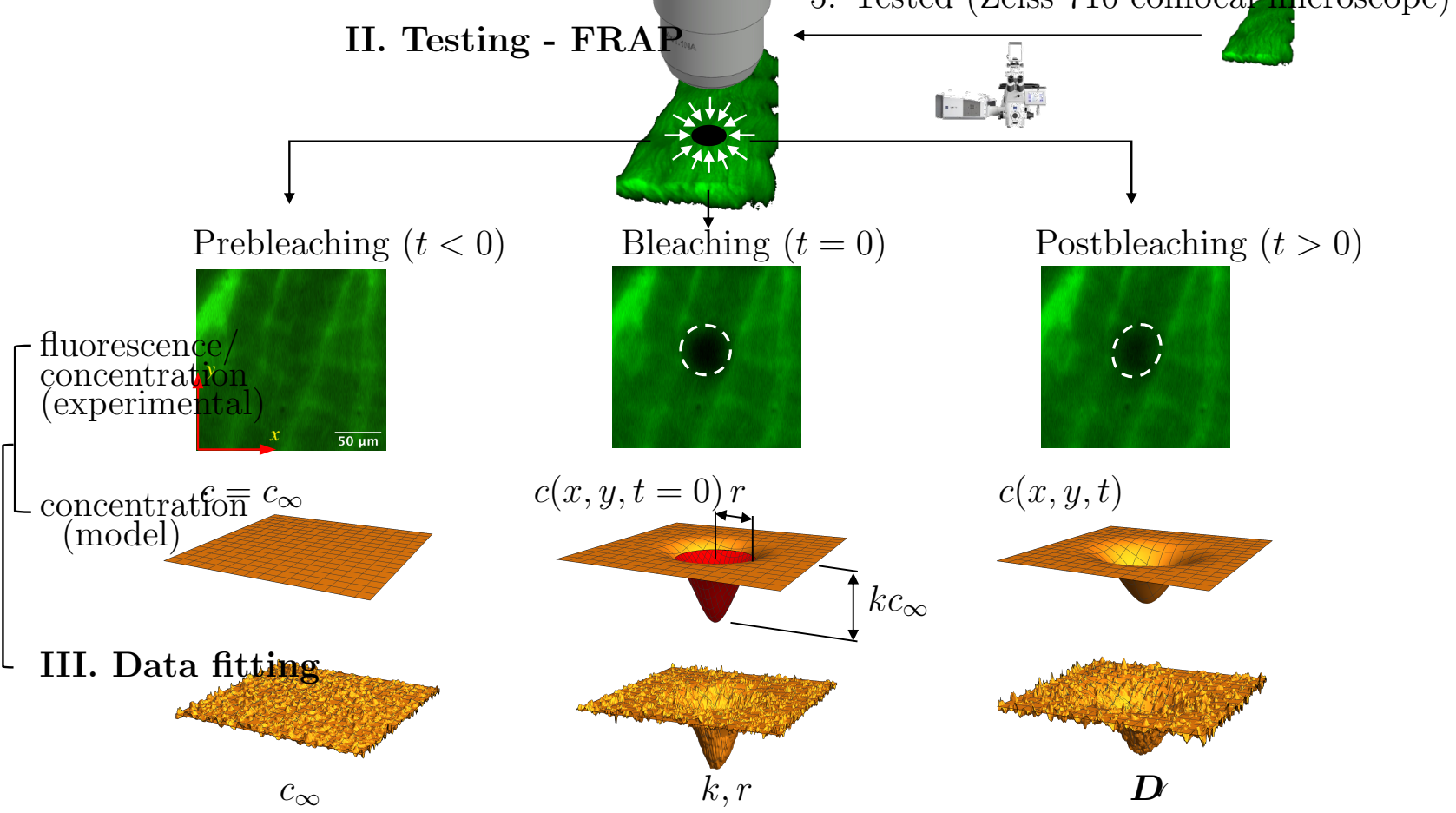

Figure 3: Schematic diagram showing the different steps followed in this work: I. tissue preparation, II. testing and III. data fitting, which were performed for select samples.

condition (4), which makes the Gaussian distribution anisotropic and allows for the application of this method to the case of the bleaching of an elliptical ROI.

Equations (1)-(4) generate a differential problem on an unbounded domain that, due to the condition (2), makes the integral $\int_{\mathbb{E}^{2}} c(\boldsymbol{x}, t) \mathrm{d} \mu(\boldsymbol{x})$ divergent (note that, here and in the sequel, $\mathrm{d} \mu(\boldsymbol{x})$ denotes the Lebesgue measure in $\mathbb{E}^{2}$ ). This inconvenience can be eliminated by noticing that the diffusion equation (1) is invariant under the transformation

$$
q(\boldsymbol{x}, t)=c_{\infty}-c(\boldsymbol{x}, t),
$$

where $q$ is an unknown function satisfying the differential problem

$$
\begin{aligned}
& \partial_{t} q=\operatorname{div}(\boldsymbol{D} \operatorname{grad} q), \\
& \lim _{\left\|\boldsymbol{x}-\boldsymbol{x}_{0}\right\| \rightarrow \infty} q(\boldsymbol{x}, t)=0, \\
& \lim _{\left\|\boldsymbol{x}-\boldsymbol{x}_{0}\right\| \rightarrow \infty}\|\operatorname{grad} q(\boldsymbol{x}, t)\|=0, \\
& q(\boldsymbol{x}, 0) \equiv q_{0}(\boldsymbol{x})=c_{\infty} k \exp \left(-2\left(\boldsymbol{x}-\boldsymbol{x}_{0}\right) \cdot \boldsymbol{A}^{-1}\left(\boldsymbol{x}-\boldsymbol{x}_{0}\right)\right),
\end{aligned}
$$

and having convergent integral $\int_{\mathbb{E}^{2}} q(\boldsymbol{x}, t) \mathrm{d} \mu(\boldsymbol{x})<+\infty$. For future use, we also introduce an equivalent expression for the initial datum, $q_{0}(\boldsymbol{x})$, which can be recast in the form

$$
q_{0}(\boldsymbol{x})=c_{\infty} \frac{B}{2 \pi \sqrt{\operatorname{det}\left(\frac{1}{4} \boldsymbol{A}\right)}} \exp \left(-\frac{1}{2}\left(\boldsymbol{x}-\boldsymbol{x}_{0}\right) \cdot\left[\frac{1}{4} \boldsymbol{A}\right]^{-1}\left(\boldsymbol{x}-\boldsymbol{x}_{0}\right)\right),
$$


where the constant $B$ (which has the physical units of area, whereas $k$ is dimensionless) is defined by

$$
B=k 2 \pi \sqrt{\operatorname{det}\left(\frac{1}{4} \boldsymbol{A}\right)} .
$$

Note that the integral of $q_{0}(\boldsymbol{x})$ over $\mathbb{E}^{2}$ is given by $c_{\infty} B$, which means that it is as if all the particles of initial (fictitious) concentration $q_{0}(\boldsymbol{x})$ were concentrated in a bounded region of $\mathbb{E}^{2}$ of area $B$. A well-established argument allows for the determination of the unknown $q$ by means of the convolution integral

$$
q(\boldsymbol{x}, t)=\int_{\mathbb{E}^{2}} G(\boldsymbol{x}-\boldsymbol{y}, t) q_{0}(\boldsymbol{y}) \mathrm{d} \mu(\boldsymbol{y}),
$$

where $G$ is referred to as Green's function of the problem defined by (9a)-(9d). We emphasise that, consistently with the conservative nature of the problem $(9 \mathrm{a})-(9 \mathrm{~d})$, it holds true that $\int_{\mathbb{E}^{2}} q(\boldsymbol{x}) \mathrm{d} \mu(\boldsymbol{x})=$ $\int_{\mathbb{E}^{2}} q_{0}(\boldsymbol{x}) \mathrm{d} \mu(\boldsymbol{x})$, i.e. the total "mass" of the molecules is conserved. When $\boldsymbol{D}$ is independent of space and time, as is the case in the present context, $G$ is given by

$$
G(\boldsymbol{x}-\boldsymbol{y}, t)=\frac{1}{2 \pi \sqrt{\operatorname{det} \boldsymbol{\Sigma}(t)}} \exp \left(-\frac{1}{2}(\boldsymbol{x}-\boldsymbol{y}) \cdot[\boldsymbol{\Sigma}(t)]^{-1}(\boldsymbol{x}-\boldsymbol{y})\right),
$$

with

$$
\boldsymbol{\Sigma}(t)=2 t \boldsymbol{D}
$$

being the covariance tensor. We recall that $G$ satisfies identically Equation (9a) and complies with the condition

$$
\lim _{t \rightarrow 0} G(\boldsymbol{x}-\boldsymbol{y}, t)=\delta(\boldsymbol{x}-\boldsymbol{y}),
$$

where $\delta(\boldsymbol{x}-\boldsymbol{y}) \equiv \delta_{\boldsymbol{y}}(\boldsymbol{x})$ is the Dirac delta distribution centred at $\boldsymbol{y}$, and the limit is to be understood in the sense of distributions. We remark that the functional expression of $G$ does not depend on whether $\boldsymbol{D}$ and, thus $[\boldsymbol{\Sigma}(t)]^{-1}$ is isotropic or anisotropic, a statement that can be verified by direct substitution of Equation (13) into (9a).

Equation (12) can be solved in the wave-vector domain, by turning the convolution into the product of the Fourier transforms of Green's function and of the initial datum, thereby obtaining

$$
Q(\boldsymbol{\xi}, t)=\mathcal{G}(\boldsymbol{\xi}, t) Q_{0}(\boldsymbol{\xi}),
$$

where $\boldsymbol{\xi}$ is the wave vector, $Q(\boldsymbol{\xi}, t), \mathcal{G}(\boldsymbol{\xi}, t)$ and $Q_{0}(\boldsymbol{\xi})$ are the Fourier transforms of the auxiliary concentration, of Green's function and of the initial datum, respectively, and they are defined as

$$
\begin{aligned}
Q(\boldsymbol{\xi}, t) & =\int_{\mathbb{E}^{2}} q(\boldsymbol{x}, t) \exp \left(-\mathrm{i} 2 \pi \boldsymbol{\xi} \cdot\left[\boldsymbol{x}-\boldsymbol{x}_{0}\right]\right) \mathrm{d} \mu(\boldsymbol{x}), \\
\mathcal{G}(\boldsymbol{\xi}, t) & =\int_{\mathbb{E}^{2}} G(\boldsymbol{x}-\boldsymbol{y}, t) \exp (-\mathrm{i} 2 \pi \boldsymbol{\xi} \cdot[\boldsymbol{x}-\boldsymbol{y}]) \mathrm{d} \mu(\boldsymbol{x}), \\
Q_{0}(\boldsymbol{\xi}) & =\int_{\mathbb{E}^{2}} q_{0}(\boldsymbol{x}) \exp \left(-\mathrm{i} 2 \pi \boldsymbol{\xi} \cdot\left[\boldsymbol{x}-\boldsymbol{x}_{0}\right]\right) \mathrm{d} \mu(\boldsymbol{x}) .
\end{aligned}
$$

A direct computation, exploiting the symmetry and positive-definiteness of tensors $\boldsymbol{\Sigma}(t)$, for $t>0$, and $\boldsymbol{A}$, the theorem of change of variables, the Gaussian structure of $G(\boldsymbol{x}-\boldsymbol{y}, t)$ and $q_{0}(\boldsymbol{x})$, and the completing-the-square technique, yields the expressions

$$
\begin{aligned}
& \mathcal{G}(\boldsymbol{\xi}, t)=\exp \left(-4 \pi^{2} t \boldsymbol{\xi} \cdot \boldsymbol{D} \boldsymbol{\xi}\right), \\
& Q_{0}(\boldsymbol{\xi})=\frac{1}{2} \pi c_{\infty} k \sqrt{\operatorname{det} \boldsymbol{A}} \exp \left(-\frac{1}{2} \pi^{2} \boldsymbol{\xi} \cdot \boldsymbol{A} \boldsymbol{\xi}\right) .
\end{aligned}
$$

We note that [16] obtained (18a) in terms of the Fourier transform of the concentration $c$ and used it to fit experimental diffusion data and to determine the anisotropic diffusion tensor. Substitution of (18a) 
and (18b) into (16) leads to the expression of the Fourier transform of the auxiliary concentration, i.e.,

$$
\begin{aligned}
Q(\boldsymbol{\xi}, t) & =\mathcal{G}(\boldsymbol{\xi}, t) Q_{0}(\boldsymbol{\xi}) \\
& =\frac{1}{2} \pi c_{\infty} k \sqrt{\operatorname{det} \boldsymbol{A}} \exp \left(-\frac{1}{2} \pi^{2} \boldsymbol{\xi} \cdot[8 \boldsymbol{D} t+\boldsymbol{A}] \boldsymbol{\xi}\right) .
\end{aligned}
$$

Upon setting $\boldsymbol{R}(t):=8 \boldsymbol{D} t+\boldsymbol{A}$, and noticing that tensor $\boldsymbol{R}(t)$ is symmetric and positive definite for all $t \geq 0, q(\boldsymbol{x}, t)$ can be found by performing the inverse Fourier transform of $Q(\boldsymbol{\xi}, t)$, i.e.,

$$
\begin{aligned}
q(\boldsymbol{x}, t) & =\int_{\mathbb{K}^{2}} Q(\boldsymbol{\xi}, t) \exp \left(\mathrm{i} 2 \pi \boldsymbol{\xi} \cdot\left[\boldsymbol{x}-\boldsymbol{x}_{0}\right]\right) \mathrm{d} \mu(\boldsymbol{\xi}) \\
& =c_{\infty} k \frac{\sqrt{\operatorname{det} \boldsymbol{A}}}{\sqrt{\operatorname{det} \boldsymbol{R}(t)}} \exp \left(-2\left(\boldsymbol{x}-\boldsymbol{x}_{0}\right) \cdot[\boldsymbol{R}(t)]^{-1}\left(\boldsymbol{x}-\boldsymbol{x}_{0}\right)\right),
\end{aligned}
$$

where $\mathbb{K}^{2}$ is the space of wave vectors $\boldsymbol{\xi}$. We notice that, for $t=0$, it holds that $\boldsymbol{R}(0)=\boldsymbol{A}$ and Equation (20) returns $q_{0}(\boldsymbol{x})$. Finally, the concentration $c(\boldsymbol{x}, t)$ reads

$$
\begin{aligned}
c(\boldsymbol{x}, t) & =c_{\infty}-q(\boldsymbol{x}, t) \\
& =c_{\infty}\left[1-k \frac{\sqrt{\operatorname{det} \boldsymbol{A}}}{\sqrt{\operatorname{det} \boldsymbol{R}(t)}} \exp \left(-2\left(\boldsymbol{x}-\boldsymbol{x}_{0}\right) \cdot[\boldsymbol{R}(t)]^{-1}\left(\boldsymbol{x}-\boldsymbol{x}_{0}\right)\right)\right] .
\end{aligned}
$$

Remark 2. All the calculations leading to Equations (18a), (18b) and (20) are analytical. More importantly, we emphasize that they hold true regardless of whether the tensors featuring in the Gaussian integrals are isotropic or anisotropic. As a sketch of such calculations, we look at the determination of $Q_{0}(\boldsymbol{\xi})$ in (18b). Specifically, we perform the change of variables $\boldsymbol{z}-\boldsymbol{x}_{0}=\boldsymbol{A}^{-1 / 2}(\boldsymbol{x}-$ $\left.\boldsymbol{x}_{0}\right)$, which entails the change of measure $\mathrm{d} \mu(\boldsymbol{z})=\operatorname{det}\left(\boldsymbol{A}^{-1 / 2}\right) \mathrm{d} \mu(\boldsymbol{x})=(\operatorname{det} \boldsymbol{A})^{-1 / 2} \mathrm{~d} \mu(\boldsymbol{x})$, and we rewrite Equation $(17 \mathrm{c})$

$$
\begin{aligned}
Q_{0}(\boldsymbol{\xi}) & =\int_{\mathbb{E}^{2}} q_{0}(\boldsymbol{x}) \exp \left(-\mathrm{i} 2 \pi \boldsymbol{\xi} \cdot\left[\boldsymbol{x}-\boldsymbol{x}_{0}\right]\right) \mathrm{d} \mu(\boldsymbol{x}) \\
& =c_{\infty} k \sqrt{\operatorname{det} \boldsymbol{A}} \int_{\mathbb{E}^{2}} \mathrm{e}^{-2\left\|\boldsymbol{z}-\boldsymbol{x}_{0}\right\|^{2}-\mathrm{i} 2 \pi \boldsymbol{A}^{1 / 2} \boldsymbol{\xi} \cdot\left[\boldsymbol{z}-\boldsymbol{x}_{0}\right]} \mathrm{d} \mu(\boldsymbol{z}) \\
& =c_{\infty} k \sqrt{\operatorname{det} \boldsymbol{A}} \mathrm{e}^{-\frac{1}{2} \pi^{2} \boldsymbol{\xi} \cdot \boldsymbol{A} \boldsymbol{\xi}} \int_{\mathbb{E}^{2}} \mathrm{e}^{-\left[\sqrt{2}\left(\boldsymbol{z}-\boldsymbol{x}_{0}\right)+\mathrm{i} \frac{2 \pi \boldsymbol{A}^{1 / 2} \boldsymbol{\xi}}{2 \sqrt{2}}\right]^{2}} \mathrm{~d} \mu(\boldsymbol{z}) .
\end{aligned}
$$

Finally, since the integral on the very far right-hand-side of Equation (22) is Gaussian and equal to

$$
\int_{\mathbb{E}^{2}} \mathrm{e}^{-\left[\sqrt{2}\left(\boldsymbol{z}-\boldsymbol{x}_{0}\right)+\mathrm{i} \frac{2 \pi \boldsymbol{A}^{1 / 2} \boldsymbol{\xi}}{2 \sqrt{2}}\right]^{2}} \mathrm{~d} \mu(\boldsymbol{z})=\frac{1}{2} \pi,
$$

one obtains the result reported in Equation (18b), i.e.,

$$
Q_{0}(\boldsymbol{\xi})=\frac{1}{2} \pi c_{\infty} k \sqrt{\operatorname{det} \boldsymbol{A}} \exp \left(-\frac{1}{2} \pi^{2} \boldsymbol{\xi} \cdot \boldsymbol{A} \boldsymbol{\xi}\right) .
$$

Remark 3. In the case in which both $\boldsymbol{D}$ and $\boldsymbol{A}$ are isotropic tensors, i.e., $\boldsymbol{D}=D \boldsymbol{I}$ and $\boldsymbol{A}=r^{2} \boldsymbol{I}$, where $\boldsymbol{I}$ is the identity tensor and $r$ is the effective radius of the bleached area, which is now circular, Equation (21) becomes

$$
c_{\text {iso }}(\boldsymbol{x}, t)=c_{\infty}\left[1-k \frac{r^{2}}{8 D t+r^{2}} \exp \left(-\frac{2\left\|\boldsymbol{x}-\boldsymbol{x}_{0}\right\|^{2}}{8 D t+r^{2}}\right)\right] .
$$




\subsection{Data Fitting}

In this section, we briefly describe the procedure for obtaining the components of the diffusivity tensor from FRAP experiments and, in Section 4, we clarify the procedure with examples. The fitting is performed over three steps:

1. To remove possible unevenness in the illumination, at each time $t$, the measured fluorescence intensity $I(\boldsymbol{x}, t)$ of the image is first normalised with respect to the intensity $I_{\mathrm{p}}(\boldsymbol{x})$ of a prebleached image (see for example [6], in which the normalised fluorescence intensity $\bar{I}(\boldsymbol{x}, t)$ is termed "relative fluorescence intensity"), i.e., the normalised intensity is given by

$$
\bar{I}(\boldsymbol{x}, t)=\frac{I(\boldsymbol{x}, t)}{I_{\mathrm{p}}(\boldsymbol{x})},
$$

and, for ideal conditions, represents the normalised concentration $\bar{c}(\boldsymbol{x}, t)=\bar{c}(\boldsymbol{x}, t) / c_{\infty}$. In [6], it is assumed that $\bar{I}(\boldsymbol{x}, t)$ is related to $\bar{c}(\boldsymbol{x}, t)$ by $\bar{I}(\boldsymbol{x}, t)=\beta(t) \bar{c}(\boldsymbol{x}, t)$, where $\beta(t)$ is a parameter that accounts for variations of intensity with time, such as drifting and fluctuations in light intensity.

2. In a given FRAP experiment, parameters $k$ and $\boldsymbol{A}$ are fixed throughout. These parameters are obtained from fitting the experimental data of the bleached image, represented by $\bar{I}(\boldsymbol{x}, 0)$, with the initial condition, $\bar{c}(\boldsymbol{x}, 0)=c(\boldsymbol{x}, 0) / c_{\infty}$, the normalised form of Equation (4). All calculations were performed using Mathematica (Wolfram Research, Champaign, Illinois, USA). The nonlinear solver NonlinearModelFit was used to fit $k$ and the components $A_{11}, A_{22}$ and $A_{12}$ of the symmetric tensor $\boldsymbol{A}$. The QuasiNewton method was used for all calculations.

3. At each postbleached frame, fitting of normalised intensity $\bar{I}(\boldsymbol{x}, t)$ is performed with the normalised form of Equation (21), i.e., $\bar{c}(\boldsymbol{x}, t)$, with the fitting parameters being the components $D_{11}, D_{22}$ and $D_{12}$ of the diffusivity tensor $\boldsymbol{D}$. Finally, effective values of the diffusivities can be obtained as the mean values of the fitted diffusivities with time. We will discuss an additional approach for obtaining the effective measures of the fitted diffusivities based on the use of the moments of inertia in Section 6.

\section{Anisotropic Diffusion in Ligaments}

In this section, we validate the approach by examining the diffusion anisotropy ratio for $3 \mathrm{kDa}-\mathrm{FITC}$ conjugated dextran in porcine medial collateral ligaments (MCL), and compare the ratio to that obtained by [11].

\subsection{Preparation}

This study was carried out in accordance with the guidelines of the Canadian Council of Animal Care and was approved by the committee for Animal Use and Ethics at the University of Calgary. Fresh knee joints of young pigs (3-6) month-old were obtained from a local abattoir. Full thickness MCL tissues were collected from the midsection of the MCL, to ensure presence of fibres with strong alignment. Thin samples (Figure 4) of the tissues with $70 \mu \mathrm{m}$ thickness were then cut using a Leica CM3050 S cryostat. Three sample thicknesses were initially considered: 30, 50 and $70 \mu \mathrm{m}$. We noticed that with the $30 \mu \mathrm{m}$ and $50 \mu \mathrm{m}$ sections, full recovery from FRAP tests did not always occur, while in the $70 \mu \mathrm{m}$, full recovery always occurred. This might be related to the amount of fluid that can be retained by the fibre network. Also, there might have been disruptions in the fibre network in the thin samples. The samples adhered properly to the coverslips, and were kept hydrated with phosphate buffered saline (PBS) until the stain was applied. Afterwards, samples were stained with solution of PBS and $3 \mathrm{kDa}$ FITC-Dextran (Sigma-Aldrich, Missouri, United States) with a concentration of $2 \mathrm{mg} / \mathrm{ml}(0.67 \mathrm{mM})$. Samples were stained at $4^{\circ} \mathrm{C}$ for 24 hours. Given the thickness of the samples, full penetration of the stain was possible during the staining time. Prior to the FRAP tests, the stained 
samples were stored in a humidity chamber to keep them hydrated, and also to prevent exposure to natural light and, thus, bleaching.

Remark 4. Preliminary tests were performed on full thickness-ligaments, without sectioning and, later, sectioned ligaments were used. There are several advantages in using thin compared to thick ligament samples. First, in thin samples, scar tissue is avoided and illumination is even. Second, thin ligament samples adhere to the coverslip more easily, providing better stability for FRAP tests. Third, the fluorescence recovery from the off-plane direction is minimised, providing a more accurate measure of the in-plane diffusivity tensor.
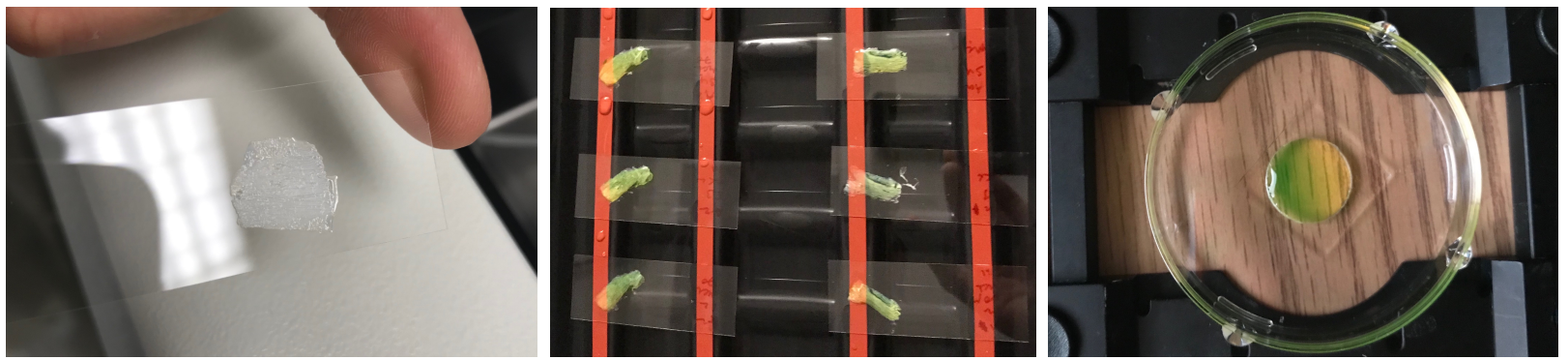

Figure 4: An MCL section (left), a set of stained MCL sections, and a solution of 3 kDa FITCdextran and PBS, placed on a glass bottom dish to minimise fluid drift during the FRAP experiments.

\subsection{Testing}

An inverted microscope (Zeiss LSM 710, Germany) with a $40 \times / 1.1$ NA water-immersion objective (Zeiss Inc., Germany) was used for both imaging and bleaching. An Argon laser of $25 \mathrm{~mW}$ was used for all testing. Imaging was performed at $\sim 0.5 \%$ laser power, and bleaching at $100 \%$. The field of view was $212 \mu \mathrm{m} \times 212 \mu \mathrm{m}$ (pixel size: $0.42 \mu \mathrm{m}$ ). The ROI diameter was set to $42 \mu \mathrm{m}$ for all experiments (100 pixels). The excitation maximum of $3 \mathrm{kDa}$ FITC-Dextran stain is $490 \mathrm{~nm}$ and, therefore, the laser wavelength was set at $488 \mathrm{~nm}$ for both imaging and bleaching. Emitted light was collected above $520 \mathrm{~nm}$. Since the theory adopted in this work is based on fluorescence recovery in the plane, and neglects any contribution from the off-plane direction, ideally bleaching should be performed throughout the thickness of the sample, in order to prevent off-plane recovery [?, as done, e.g., by]]greene2008changes. However, in order to avoid the cut-surfaces and any resulting disruption in the fibre network, bleaching was performed in an optical slice smaller than the sample's thickness. A set of FRAP tests was initially performed using two pinhole openings: $265 \mu \mathrm{m}$ which results in a $\sim 7 \mu \mathrm{m}$ optical slice, and $520 \mu \mathrm{m}$, which results in $\mathrm{a} \sim 14 \mu \mathrm{m}$ optical slice. The difference between the results was not significant, indicating that the planar recovery was not sensitive to the off-plane direction.

We note here that the ROI diameter, $42 \mu \mathrm{m}$, was chosen to be large enough to be representative of the tissue, and to capture the anisotropy, as can be seen in the scanning electron microscopy images in Figure 1, in which the fibre orientation is visible within a region smaller than $20 \mu \mathrm{m}$, whereas our ROI diameter is $42 \mu \mathrm{m}$, i.e., the anisotropy is generally captured in the FRAP experiments. The ROI was also chosen to be small enough in order to be approximated as homogeneous. We initially studied the diffusivity for different ROI sizes, and adopted an intermediate size, using the same microscope objective, for the following reasons

1. Bleaching a smaller ROI than the one chosen in our experiments, leads to recovery during bleaching, as the half-life recovery time is proportional to the squared diameter of the bleached ROI. This could lead to underestimating the diffusivity measurement;

2. Increasing the ROI beyond the $42 \mu \mathrm{m}$ diameter ROI chosen in our experiments would require a longer scanning interval of the post-bleached frames, leading to unwanted photo-bleaching. 
Moreover, as the size of the ROI increases, while using the same microscope objective, the accuracy of the numerical fitting would be compromised as will be explained in the COMSOL simulations in Section 5.

In order to quantify the effect of off-plane recovery, a set of independent FRAP tests was performed using a solution of $3 \mathrm{kDa}$ FITC-dextran and PBS, at the same concentration as used in the experiments with ligament samples, at a $\sim 14 \mu \mathrm{m}$ optical slice thickness, and similar experimental conditions as for the ligament tests. The results were then compared to a theoretical baseline, represented by the Stokes-Einstein equation, as will be explained in Section 4.3.

\subsection{Results and Analysis}

In the following, we report the results of diffusivity magnitude and anisotropy ratio for $3 \times 3 \times 15$ FRAP tests (performed on 3 animals, with 3 samples tested from each animal, and 15 FRAP tests performed for each sample; the representation of an example set of data is given in Figure 5). The average diffusivity $D=\frac{1}{2} \operatorname{tr} \boldsymbol{D}$ (coefficient of the spherical part of the two-dimensional diffusivity tensor) was $90.2 \pm 1.13 \mu \mathrm{m}^{2} / \mathrm{s}(\mathrm{SE})$, and the anisotropy ratio $D_{1} / D_{2}$ (where $D_{1}$ and $D_{2}$ are the maximum and minimum eigenvalues of the in-plane diffusivity tensor $\boldsymbol{D}$ ) was $1.42 \pm 0.015$ (SE). The anisotropy ratio is close to that obtained by [11], who found it to be $\sim 1.33 \pm 0.07$.
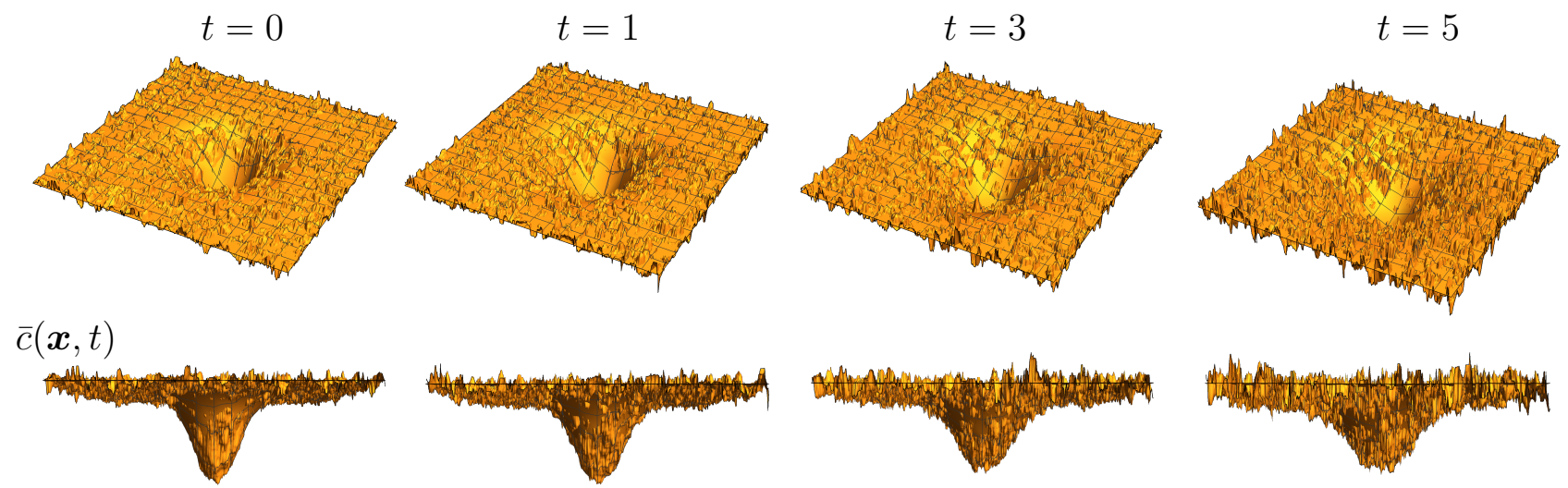

Figure 5: Example of data fitting of normalised concentration with the theoretical solution for an MCL sample, following steps 2 and 3 of the validation method in Section 4, respectively. Images show the initial condition (left) and three postbleached images.

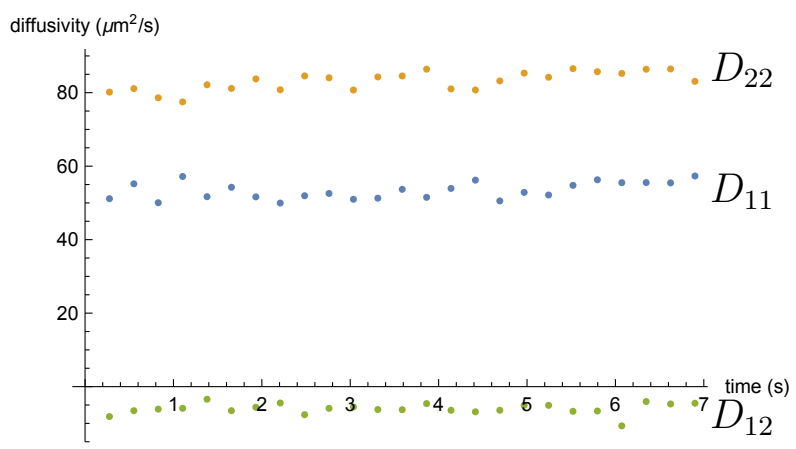

Figure 6: Diffusivity values obtained from fitting of experimental data from FRAP tests on porcine MCLs.

In Figure 6, we show the values of the diffusivity components found from fitting for each frame. In a given FRAP test, the average of the diffusivity component over time provides an effective measure for 
each component of the diffusivity tensor. However, one way of calculating the effective diffusivity over time is through the moment of inertia tensor (or, simply, tensor of inertia) $\boldsymbol{M}$ of the concentration $c$, i.e.,

$$
\boldsymbol{M}(t)=\int_{\mathbb{E}^{2}} c(\boldsymbol{x}, t)\left[\left\|\boldsymbol{x}-\boldsymbol{x}_{0}\right\|^{2} \boldsymbol{I}-\left(\boldsymbol{x}-\boldsymbol{x}_{0}\right) \otimes\left(\boldsymbol{x}-\boldsymbol{x}_{0}\right)\right] \mathrm{d} \mu(\boldsymbol{x}),
$$

where $\boldsymbol{I}$ is the identity tensor. The integral in (27) diverges because the concentration $c(\cdot, t)$ tends to the non-zero value $c_{\infty}$ at infinity. Thus, we use the rescaled tensor of inertia $\boldsymbol{J}$ of the quantity $q(\cdot, t)=c_{\infty}-c(\cdot, t)$ defined in Equation (8), which instead converges to zero at infinity, i.e.,

$$
\boldsymbol{J}(t)=\int_{\mathbb{E}^{2}} q(\boldsymbol{x}, t)\left[\left\|\boldsymbol{x}-\boldsymbol{x}_{0}\right\|^{2} \boldsymbol{I}-\left(\boldsymbol{x}-\boldsymbol{x}_{0}\right) \otimes\left(\boldsymbol{x}-\boldsymbol{x}_{0}\right)\right] \mathrm{d} \mu(\boldsymbol{x}) .
$$

To determine an explicit expression of $\boldsymbol{J}(t)$, we exploit the well-established property of Gaussian integrals. To begin with, we notice that for every symmetric second-order tensor $\boldsymbol{H}$ with positive determinant, the following integral identity holds true

$$
\begin{aligned}
\Im(\boldsymbol{H}) & :=\int_{\mathbb{E}^{2}} \exp \left(-2\left(\boldsymbol{x}-\boldsymbol{x}_{0}\right) \cdot \boldsymbol{H}^{-1}\left(\boldsymbol{x}-\boldsymbol{x}_{0}\right)\right) \mathrm{d} \mu(\boldsymbol{x}) \\
& =\int_{\mathbb{E}^{2}} \exp \left(-2 \boldsymbol{H}^{-1}:\left[\left(\boldsymbol{x}-\boldsymbol{x}_{0}\right) \otimes\left(\boldsymbol{x}-\boldsymbol{x}_{0}\right)\right]\right) \mathrm{d} \mu(\boldsymbol{x}) \\
& =\frac{1}{2} \pi \sqrt{\operatorname{det} \boldsymbol{H}} .
\end{aligned}
$$

Equation (29) completely defines $\mathfrak{I}$ as a differentiable scalar function of $\boldsymbol{H}$, and differentiation of its expression on the far right-hand-side of (29) yields

$$
\frac{\partial \mathfrak{I}}{\partial \boldsymbol{H}}(\boldsymbol{H})=\frac{1}{4} \pi \sqrt{\operatorname{det} \boldsymbol{H}} \boldsymbol{H}^{-1} .
$$

On the other hand, by differentiating the integral expression of $\mathfrak{I}$ with respect to $\boldsymbol{H}$, and employing the tensor identity

$$
\begin{aligned}
\frac{\partial \boldsymbol{H}^{-1}}{\partial \boldsymbol{H}}(\boldsymbol{H}) & =-\frac{1}{2}\left[\boldsymbol{H}^{-1} \underline{\otimes} \boldsymbol{H}^{-1}+\boldsymbol{H}^{-1} \bar{\otimes} \boldsymbol{H}^{-1}\right], \\
\frac{\partial\left(\boldsymbol{H}^{-1}\right)_{m n}}{\partial H_{i j}}(\boldsymbol{H}) & =-\frac{1}{2}\left[\left(\boldsymbol{H}^{-1}\right)_{m i}\left(\boldsymbol{H}^{-1}\right)_{n j}+\left(\boldsymbol{H}^{-1}\right)_{m j}\left(\boldsymbol{H}^{-1}\right)_{n i}\right],
\end{aligned}
$$

which makes use of the special tensor products $\underline{\otimes}$ and $\bar{\otimes}$ introduced by [4], one obtains

$$
\frac{\partial \mathfrak{I}}{\partial \boldsymbol{H}}(\boldsymbol{H})=2 \int_{\mathbb{E}^{2}} \exp \left(-2 \boldsymbol{H}^{-1}:\left[\left(\boldsymbol{x}-\boldsymbol{x}_{0}\right) \otimes\left(\boldsymbol{x}-\boldsymbol{x}_{0}\right)\right]\right)\left\{\boldsymbol{H}^{-1}\left[\left(\boldsymbol{x}-\boldsymbol{x}_{0}\right) \otimes\left(\boldsymbol{x}-\boldsymbol{x}_{0}\right)\right] \boldsymbol{H}^{-1}\right\} \mathrm{d} \mu(\boldsymbol{x}) .
$$

$$
\frac{\partial \mathfrak{I}}{\partial \boldsymbol{H}}(\boldsymbol{H})=\boldsymbol{H}^{-1}\left\{2 \int_{\mathbb{E}^{2}} \exp \left(-2 \boldsymbol{H}^{-1}:\left[\left(\boldsymbol{x}-\boldsymbol{x}_{0}\right) \otimes\left(\boldsymbol{x}-\boldsymbol{x}_{0}\right)\right]\right)\left[\left(\boldsymbol{x}-\boldsymbol{x}_{0}\right) \otimes\left(\boldsymbol{x}-\boldsymbol{x}_{0}\right)\right] \mathrm{d} \mu(\boldsymbol{x})\right\} \boldsymbol{H}^{-1} .
$$

Hence, equating (30) to (33) and multiplying the resulting expression from the left and the right by $\boldsymbol{H}$, one obtains

$$
\int_{\mathbb{E}^{2}} \exp \left(-2 \boldsymbol{H}^{-1}:\left[\left(\boldsymbol{x}-\boldsymbol{x}_{0}\right) \otimes\left(\boldsymbol{x}-\boldsymbol{x}_{0}\right)\right]\right)\left[\left(\boldsymbol{x}-\boldsymbol{x}_{0}\right) \otimes\left(\boldsymbol{x}-\boldsymbol{x}_{0}\right)\right] \mathrm{d} \mu(\boldsymbol{x})=\frac{1}{8} \pi \sqrt{\operatorname{det} \boldsymbol{H}} \boldsymbol{H} .
$$

Before going further, we notice that, even though $\left(\boldsymbol{x}-\boldsymbol{x}_{0}\right) \otimes\left(\boldsymbol{x}-\boldsymbol{x}_{0}\right)$ is a singular tensor, the integral in Equation (33) is a non-singular tensor. 
By taking the trace of Equation (34), one obtains

$$
\int_{\mathbb{E}^{2}} \exp \left(-2 \boldsymbol{H}^{-1}:\left[\left(\boldsymbol{x}-\boldsymbol{x}_{0}\right) \otimes\left(\boldsymbol{x}-\boldsymbol{x}_{0}\right)\right]\right)\left\|\boldsymbol{x}-\boldsymbol{x}_{0}\right\|^{2} \mathrm{~d} \mu(\boldsymbol{x})=\frac{1}{8} \pi \sqrt{\operatorname{det} \boldsymbol{H}} \operatorname{tr} \boldsymbol{H} .
$$

Finally, by combining (35) with (34), with $\boldsymbol{H}$ replaced by $\boldsymbol{R}(t)$, and recalling the definitions of $q$ and $\boldsymbol{J}$, one finds

$$
\boldsymbol{J}(t)=\frac{1}{8} \pi c_{\infty} \sqrt{\operatorname{det} \boldsymbol{A}}\{[\operatorname{tr} \boldsymbol{R}(t)] \boldsymbol{I}-\boldsymbol{R}(t)\} .
$$

From Equation (36) and the definition of $\boldsymbol{R}(t)=8 \boldsymbol{D} t+\boldsymbol{A}$, we can see that the tensor of inertia $\boldsymbol{J}(t)$ is affine in the diffusivity tensor. More specifically, in a coordinate representation, each component of the matrix associated with $\boldsymbol{J}(t)$ is affine in one diffusivity component; i.e., each component of the matrix of $\boldsymbol{J}(t)$ is given by a linear function in one of the diffusivity components plus a constant. Indeed, $J_{11}$ is affine in $D_{22}, J_{22}$ in $D_{11}$ and $J_{12}$ in $D_{12}$. Indeed, in components, we can write

$$
[J(t)]=\frac{1}{4} c_{\infty} B\left[\begin{array}{cc}
8 D_{22} t+A_{22} & -8 D_{12} t-A_{12} \\
-8 D_{12} t-A_{12} & 8 t D_{11}+A_{11}
\end{array}\right] .
$$

For a given FRAP test, the fitted values of a component of the diffusivity tensor, at different frames, can be substituted in the expression of the tensor of inertia component that they feature in, and a best fit line can be used to find the effective diffusivity. For the example shown in Figure 6, the values of the fitted diffusivities were substituted into Equation (37), and the best fit lines are shown in Figure 7. After obtaining the matrix of the effective values of the components of the diffusivity tensor, $[D]_{\text {eff }}$, the eigenvalues and eigenvectors are found, i.e., anisotropy ratio and principal directions are known (See the sample test in Figure 8, showing the principal direction associated with the maximum eigenvalue).

It is important to point out that there are variations in the fibre alignment and fibre density, even within the same ligament sample, which contributes to variations in the diffusivity tensor and, thus, leads to relatively large standard errors. We demonstrate these structural variations using two examples. In the first case, we can see different patterns of fibre alignment, or waviness, within a few hundreds of micrometers within the same sample (Figure 9). In the second example (Figure 10), we show two explant samples collected from the same MCL tissue, with one (right) having strongly aligned fibres and higher fibre density than the other sample (left).

In order to capture inhomogeneities of the tissue, FRAP tests were carried out at different locations within the same sample. Moreover, second harmonic generation (SHG) images were collected, for selected samples, at different locations within the same sample, and for different samples using twophoton excitation microscopy (FVMPE-RS, Olympus, Japan), using a 25×/1.05 NA water-immersion objective (Olympus Inc., Japan). However, since the FRAP testing was performed using confocal microscopy, for which the theory is developed, and the SHG images were obtained by two-photon excitation microscopy, FRAP tests could not be matched to the SHG images.

In order to assess the effect of the off-plane recovery, we first discuss the theoretical measure for diffusivity for a spherical particle with radius $r$, in a dilute solution and a small Reynold's number, which is given by the Stokes-Einstein equation [?, see, e.g.,]]brinkman1949calculation

$$
D_{\mathrm{th}}=\frac{k_{\mathrm{B}} T}{6 \pi \mu r}
$$

where $k_{\mathrm{B}}=1.380648 \times 10^{-23} \mathrm{~m}^{2} \mathrm{~kg} / \mathrm{s}^{2} \mathrm{~K}$ is Boltzmann's constant, $T$ is the temperature expressed in Kelvin, $\mu$ is the dynamic viscosity of the fluid. All tests were performed at room temperature, $T=298 \mathrm{~K}$, and the solution's viscosity is assumed to be $8.9 \times 10^{-4} \mathrm{Pas}$ (water), and the hydrodynamic radius of the $3 \mathrm{kDa}$ dextran molecules is $1.4 \mathrm{~nm}$ (Sigma-Alrdich). The theoretical scalar diffusivity value, calculated from Equation (38) is $D_{\text {th }}=175 \mu \mathrm{m}^{2} / \mathrm{s}$. The spherical part of the diffusivity for $n=40$ FRAP tests was $170 \pm 1.17 \mu \mathrm{m}^{2} / \mathrm{s}$ (SE). Results suggest that an optical slice of $14 \mu \mathrm{m}$ is sufficient for assuming planar recovery. 

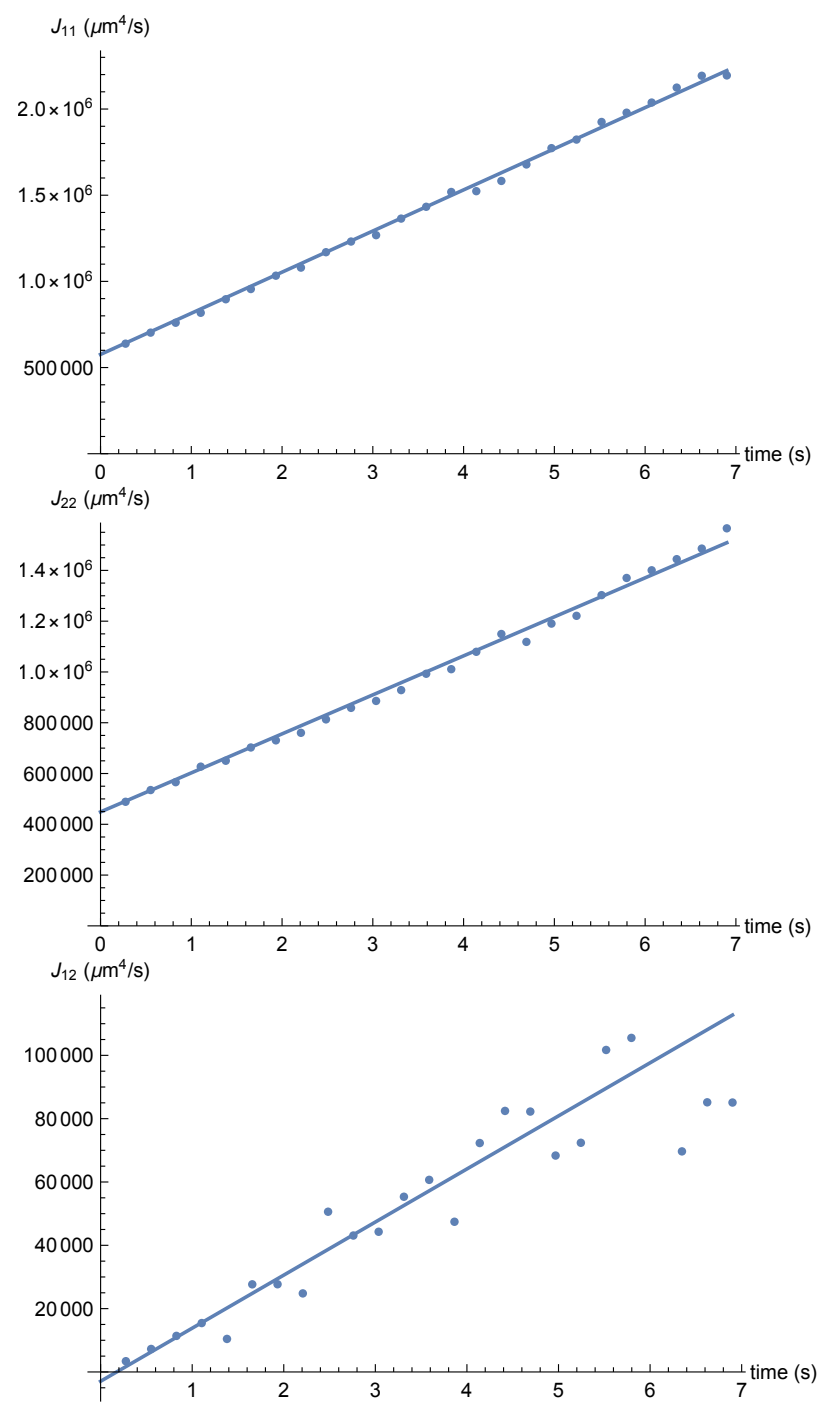

Figure 7: Moments of inertia of the different fitted diffusivities, together with the best fit lines. 


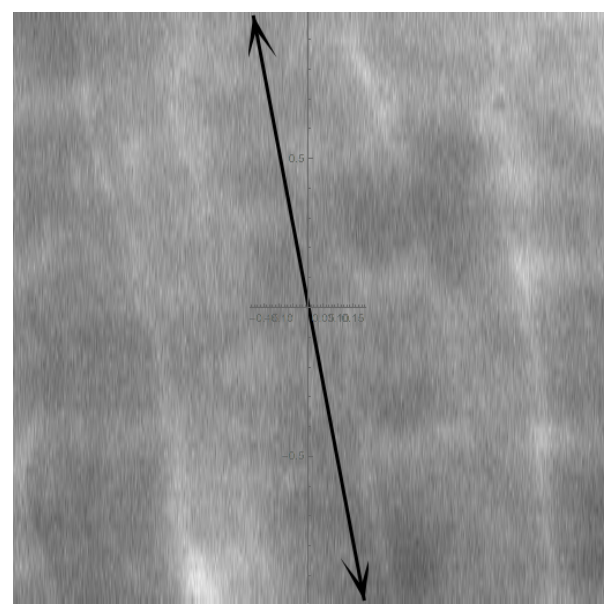

Figure 8: Image of a porcine ligament sample, showing the maximum principal direction of diffusion anisotropy found by best fitting the experimental data.

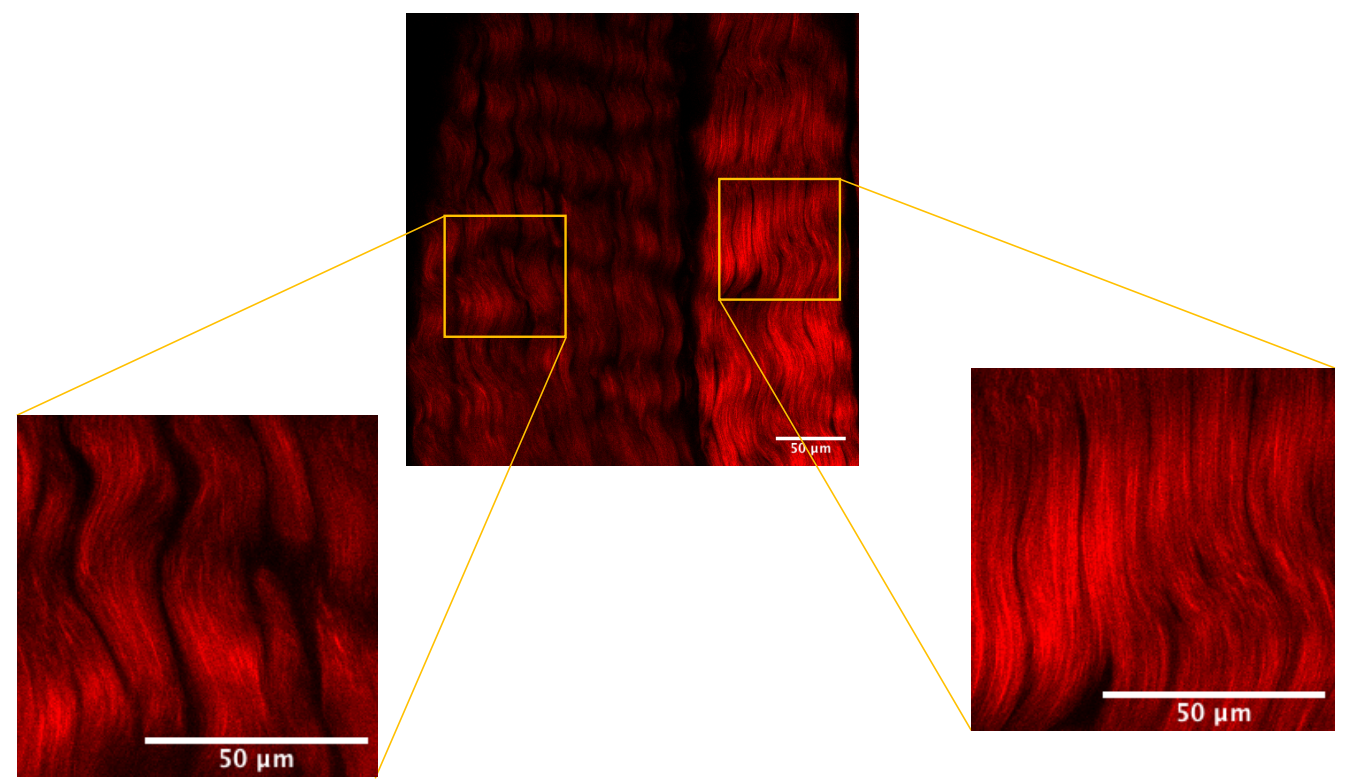

Figure 9: SHG image of an MCL sample, showing two regions with different fibre alignment within less than $300 \mu \mathrm{m}$ of each other.

\section{Error Analysis: COMSOL Simulation}

In this section, we examine the validity and the limitations of the method by numerically simulating an anisotropic FRAP experiment. The simulation serves two purposes

1. A comparison between the analytical solution used for fitting Equation (21), which assumes the condition at infinity $\lim _{\left\|\boldsymbol{x}-\boldsymbol{x}_{0}\right\| \rightarrow \infty} c(\boldsymbol{x}, t)=c_{\infty}$, and the solution of the diffusion equation (1) for a bounded domain, represented by the dimensions of the frames. The error is then plotted as a function of time to determine a time window through which the error can be tolerated. We should emphasize at this point that data fitting is done with respect to the analytical solution of the unbounded domain and, therefore, the error resulting from the comparison with the bounded domain case should be regarded as the worst case scenario, which one would not expect to occur in reality. As for the dimensions of the frame, a $212 \times 212 \mu \mathrm{m}^{2}$ window was used throughout the simulation, which corresponds to the dimensions used in the experiments.

2. Effect of parameters $k$ and $\boldsymbol{A}$ on the error. For simplicity, the spherical part of $\boldsymbol{A}$, i.e., $r^{2}$ (or alternatively, of $r$ ), is considered. The parameters chosen for $k$ and $r$ were based on the 

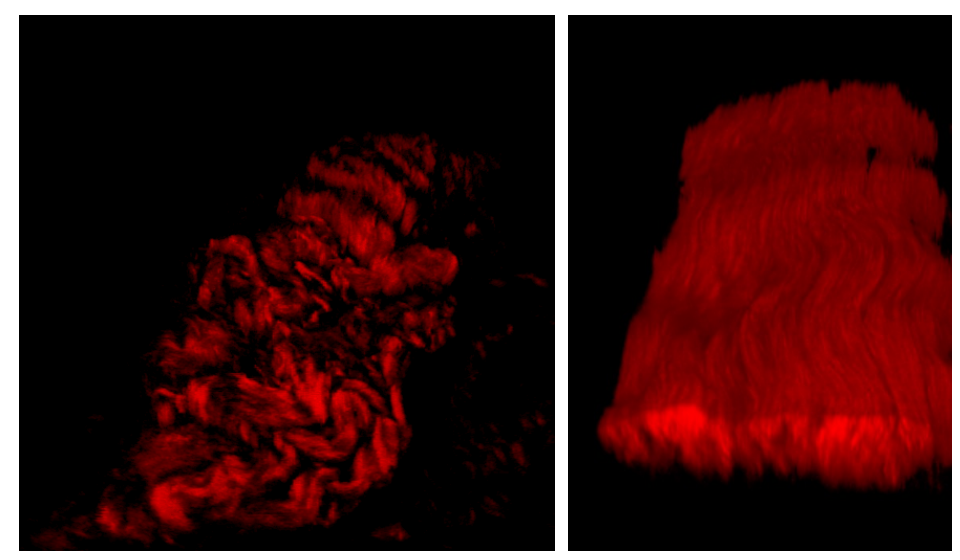

Figure 10: SHG images taken from two samples of the same MCL tissue, showing significant differences in fibre alignment and fibre density.

experiments in Section 4, and helped in determining the level of the bleaching intensity and the radius of bleaching that can be safely used. Moreover, the diffusivity tensor used was based on the results obtained for the MCL tissues in Section 4.

Equation (1) is solved numerically on the bounded domain $\Omega=[-L / 2, L / 2] \times[-L / 2, L / 2]$, i.e., the square with side $L=212 \mu \mathrm{m}$ (which corresponds to the dimensions of the frame) centered at $\boldsymbol{x}_{0}=\mathbf{0}$, by means of a finite element method, implemented in the commercially available software COMSOL Multiphysics $^{\mathrm{TM}}$, along with the initial condition (4) and for $c_{\infty}$ equal to unity and different values of $k$ and $r$. The numerical solution is found by imposing Dirichlet conditions on the entire boundary $\partial \Omega$ of $\Omega$. In particular, we impose

$$
c(\boldsymbol{x}, t)=c_{0}(\boldsymbol{x}) \quad \forall \boldsymbol{x} \in \partial \Omega, \quad \forall t .
$$

We remark that, for the comparison with the experiments, the numerical simulations were run only for the isotropic case. More specifically, for each computation, Lagrange cubic polynomials were chosen as interpolation functions and the space discretisation of the computational domain was done by means of unstructured grids, which changed adaptively for varying values of the radius of the bleached region. For instance, for $r=25 \mu \mathrm{m}$, the number of degrees of freedom of the problem was 123127. The time to complete the simulation was $30 \mathrm{~s}$, and the time discretisation of the problem followed an Euler backward scheme with the constant time step of $0.1 \mathrm{~s}$. We also note that the choice of the computational domain size $L$ is essentially dictated by optical constraints: we cannot choose too large of a window. If we were able to choose a very large window, then the numerical solution would be closer to the analytical solution, even maintaining the boundary condition $c(\boldsymbol{x}, t)=c_{0}(\boldsymbol{x})$, for $\boldsymbol{x} \in \partial \Omega$, because boundary effects would become negligible, and we would approach the ideal conditions of an infinite plane. Indeed, by increasing the size of the computational domain, the value of $c_{0}(\boldsymbol{x})$ on $\partial \Omega$ becomes closer to $c_{\infty}$, thereby reducing the weight of the boundary condition that is chosen for the numerical simulations. In other words, granting all the symmetry properties that the solution should have, any other boundary condition tending towards $c_{\infty}$ for $\left\|\boldsymbol{x}-\boldsymbol{x}_{0}\right\| \rightarrow \infty$ would yield similar results. However, it is also important to emphasize that the boundary condition $c(\boldsymbol{x}, t)=c_{0}(\boldsymbol{x})$, for $\boldsymbol{x} \in \partial \Omega$, is not the one that minimizes the discrepancies between the analytical solution and the numerical one, because it compels the numerical solution to be constant in time at the boundary of $\Omega$, whereas the analytical solution varies in time on the points of $\partial \Omega$ (it is, in fact, defined all over $\mathbb{E}^{2}$ and, thus, also on $\partial \Omega$ ). Note that we selected such a boundary condition on purpose, in order to make sure that our calculations were sufficiently robust. On the other hand, the discrepancies between the analytical and the numerical solutions could have been reduced by using the restriction of the analytical solution on $\partial \Omega$ as boundary condition. This would have avoided the differences between the values taken by the numerical and the analytical solution at the boundary of the frame (see e.g. Fig. 11), which arise because, in all the simulated cases, $c_{0}(\boldsymbol{x})$ does not depend on time. 
In order to quantify the discrepancy between the numerical solution, based on an integral evaluated on the bounded domain $\Omega \subset \mathbb{E}^{2}$, and the analytical solution (21), based on integrals evaluated over the whole $\mathbb{E}^{2}$ plane, we define the error

$$
\chi(\boldsymbol{x}, t):=\left|c_{\mathrm{n}}(\boldsymbol{x}, t)-c_{\mathrm{a}}(\boldsymbol{x}, t)\right|,
$$

where the subscripts "n" and "a" stand for "numerical" and "analytical", respectively, and we compute the $L^{2}$-, $H^{1}$ - and $L^{\infty}$-norm of the error, i.e. [2],

$$
\begin{aligned}
\|\chi(\boldsymbol{x}, t)\|_{L^{2}(\Omega)} & :=\frac{1}{\operatorname{Area}(\Omega)} \int_{\Omega}[\chi(\boldsymbol{x}, t)]^{2} \mathrm{~d} \mu(\boldsymbol{x}), \\
\|\chi(\boldsymbol{x}, t)\|_{H^{1}(\Omega)} & :=\frac{1}{\operatorname{Area}(\Omega)} \int_{\Omega}[\chi(\boldsymbol{x}, t)]^{2} \mathrm{~d} \mu(\boldsymbol{x})+\int_{\Omega}\|\operatorname{grad} \chi(\boldsymbol{x}, t)\|^{2} \mathrm{~d} \mu(\boldsymbol{x}), \\
\|\chi(\boldsymbol{x}, t)\|_{L^{\infty}(\Omega)} & :=\sup _{\boldsymbol{x} \in \Omega}\{\chi(\boldsymbol{x}, t)\} .
\end{aligned}
$$

An example simulation of a FRAP test using the parameters that were most frequently used, $k=0.7$ and $r=50 \mu \mathrm{m}$, and the accompanying error, are shown in Figure 11. We notice that the $L^{2}$ norm of the error is small for a relatively large time window, suggesting that fitting with Equation (21), which results in a smaller error compared to the simulation, is safe. For the analysis, we consider a time window comparable to that of the half recovery time of the molecule, for which an estimate can be found based on using the diffusivity of the molecule in pure fluid, Equation (38), in the relation by $[1]$

$$
\tau_{1 / 2}=\frac{r^{2}}{4 D} \gamma,
$$

where $\gamma$ is an experimental parameter that depends on the bleaching parameter $k$ for a beam with Gaussian intensity profile. For simplicity, we adopt the value 0.88 of the case of a beam of uniform intensity [?, called a "circular beam" in the paper by]from which we took the value of $\gamma$ ]axelrod1976mobility. In the case of diffusivity of $3 \mathrm{kDa}$ dextran in fluid, Equation (42) results in $\tau_{1 / 2}=3.14 \mathrm{~s}$ and for diffusion in ligaments, based on the diffusivity that obtained, $\tau_{1 / 2}=6.1 \mathrm{~s}$, when $r=50 \mu \mathrm{m}^{2} / \mathrm{s}$.

We should note here that the analysis in this work is performed for the $3 \mathrm{kDa}$ dextran molecules, but a similar analogy can also be used for larger molecules, for which larger time windows for the analysis would be considered (following Equation (42)), but the error should not increase, as diffusivity is smaller for the larger molecules. To test the effect of the intensity of bleaching and thus, $k$, and the
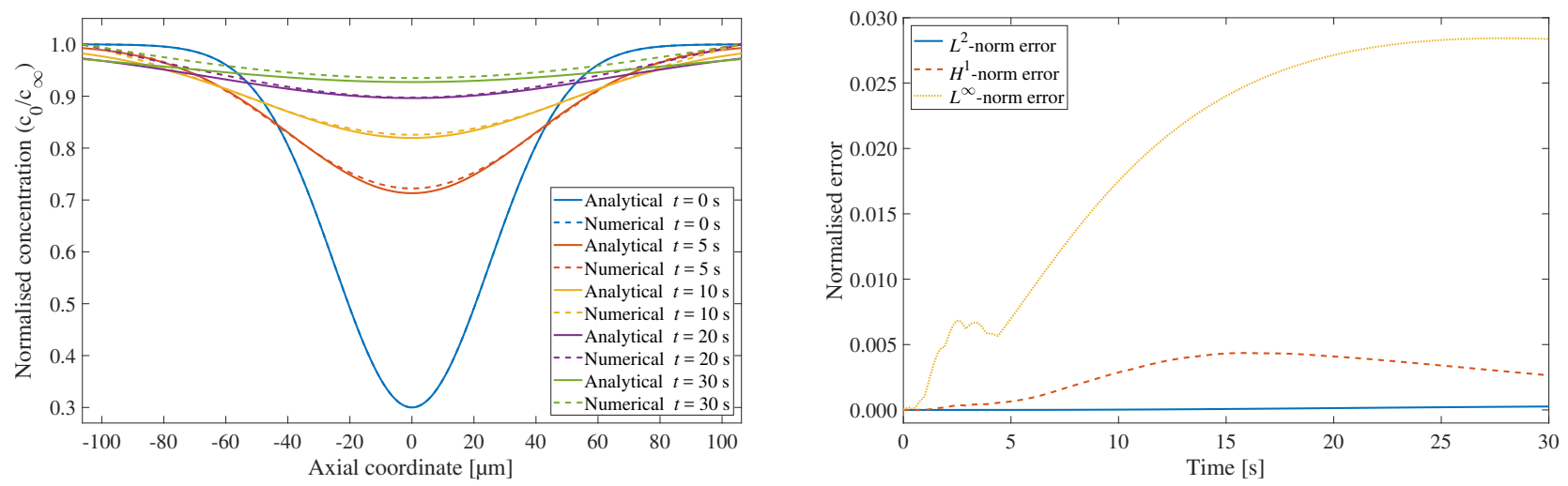

Figure 11: Concentration recovery in a simulated FRAP experiment with $k=0.7$ and $r=$ $50 \mu \mathrm{m}$, and the resulting error.

effect of the size of the bleached region and, thus, $r$, additional simulations were performed. Figure 12 shows the concentration recovery after fixing $k=0.7$, and using two values of $r: 25 \mu \mathrm{m}$ and $75 \mu \mathrm{m}$. For the case of $r=25 \mu \mathrm{m}$, we can see that the error is very small, while $r=75 \mu \mathrm{m}$ results in a much larger error. Figure 13 shows two cases, where $r$ is fixed at $50 \mu \mathrm{m}$, and two values of $k$ are considered, $k=0.5$ and 0.9 . When $k=0.5$, the error is very small, while the error for $k=0.9$ is larger. 


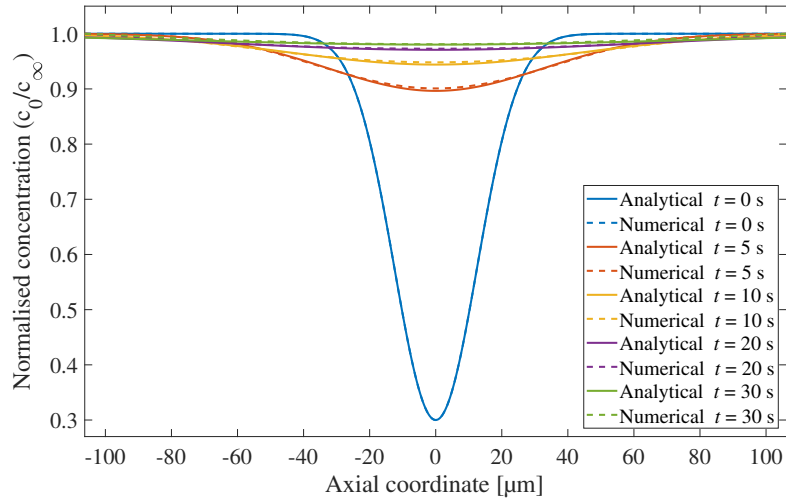
towards zero for very long times.

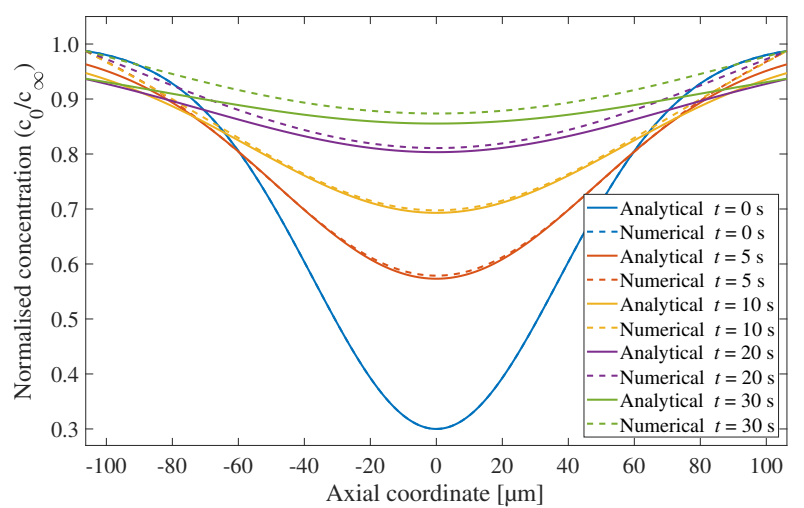

To compare the numerical solutions with the analytical ones in the isotropic case, we plot the concentration profiles for different times (left panels of Figs. 11-13) and the associated errors (right panels of Figs. 11-13). The curves on the left panels are evaluated for $x_{2}=0$ and show, thus, the concentrations as functions of $x_{1}$ only. Because of the assumed isotropy, we would obtain exactly the same curves if we evaluated the concentrations for $x_{1}=0$ and let $x_{2}$ vary in $[-L / 2, L / 2]$.

In summary, the difference between the analytical solutions (solid lines) and the numerical ones (dashed lines) can be appreciated by looking at the left panels of Figs. 11-13. The discrepancies between the numerical and the analytical curves depend on the chosen parameters $k$ and $r$. Looking at the right panels of Figs. 11-13, we notice that the $L^{2}$-norm of the error is close to zero for all times and increases for increasing values of $r$ in the time window $[0 \mathrm{~s}, 30 \mathrm{~s}]$. However, it goes towards zero for sufficiently long times. Although the same occurs also for the $H^{1}$ - and the $L^{\infty}$-norms, they exhibit larger deviations from zero in the time interval $[0 \mathrm{~s}, 30 \mathrm{~s}]$. Regarding the $H^{1}$-norm, this behaviour may be due to the discrepancies in the derivatives of the concentrations, which tend to become more evident in the neighbourhood of the domain's boundaries. In the case of the $L^{\infty}$-norm, it is possible to observe quite rapid oscillations close to $t=0 \mathrm{~s}$, followed by a more stable trend, which goes asymptotically
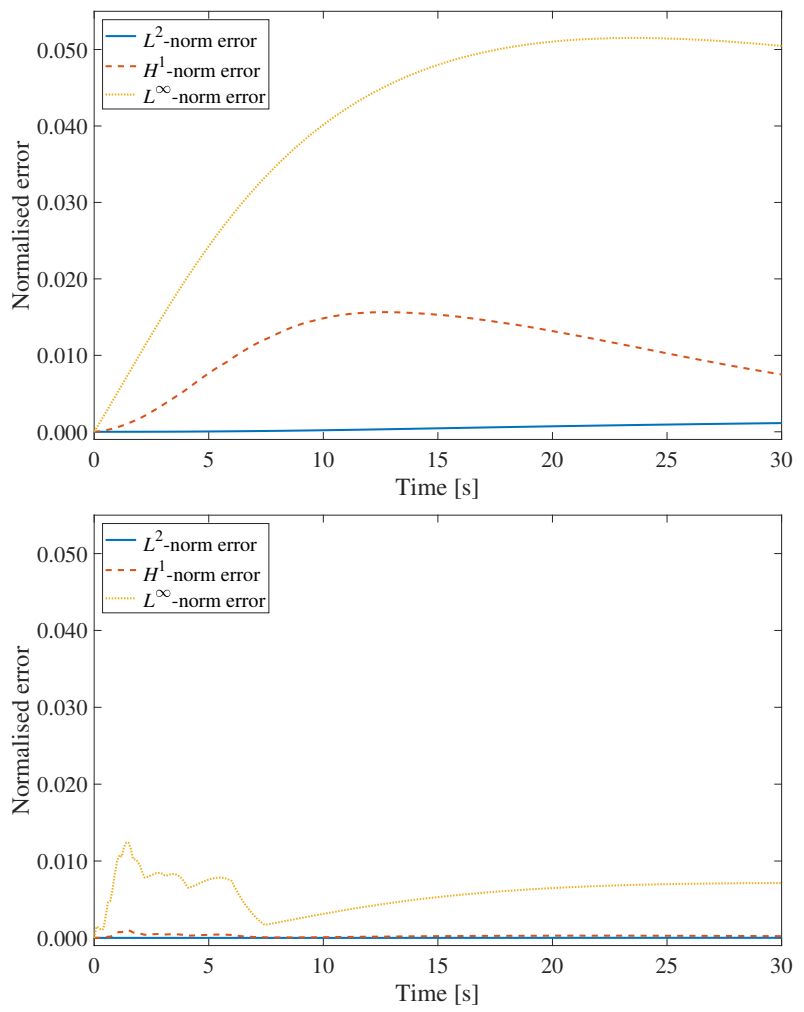

Figure 12: Effect of varying $r$ on the concentration recovery and the resulting error in a simulated FRAP experiment with $k=0.7$ for two values of $r: r=75 \mu \mathrm{m}$ (top row) and $r=25 \mu \mathrm{m}$ (bottom row).

\section{Discussion and Conclusions}

Fluorescence recovery after photobleaching is a common technique for studying the mobility of molecules on the cellular and on the tissue level. Although the emergence of multi-photon microscopy allowed for bleaching of three-dimensional ROIs and, thus, some models for multi-photon FRAP have been introduced [?, e.g.,]]mazza2008new,shi2014measurement, the theory and the analysis are generally complicated, and due to the relatively large time needed for bleaching and imaging of three-dimensional regions, the diffusivity measurement could be underestimated, e.g., due to recovery during bleaching. Therefore, studying the two-dimensional diffusion behaviour with confocal microscopes is still 

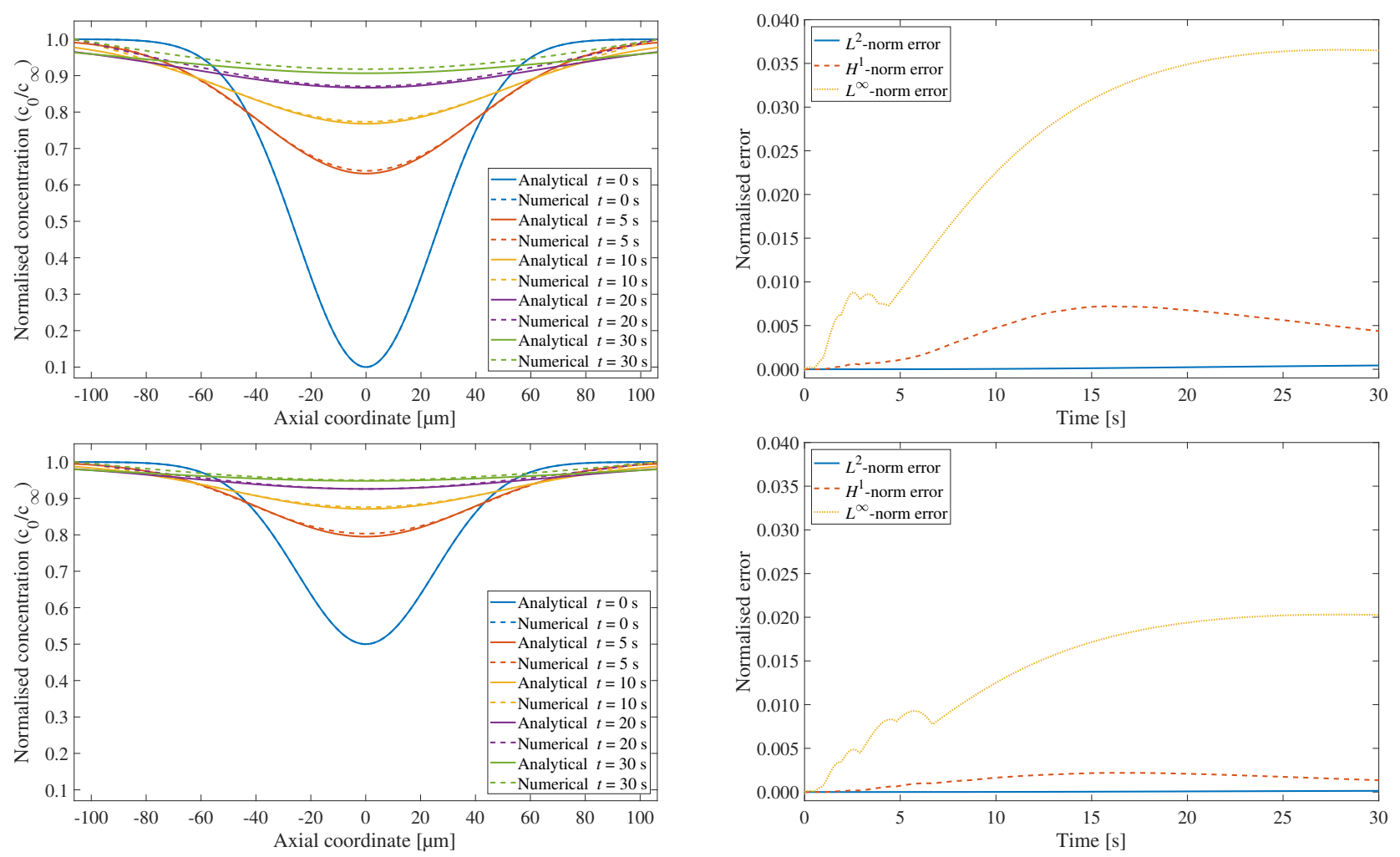

Figure 13: Effect of varying $k$ on the concentration recovery and the resulting error in a simulated FRAP experiment with $r=50 \mu \mathrm{m}$ and two values of $k: k=0.9$ (top row) and $k=0.5$ (bottom row).

widespread. Here, we propose to add an approach for quantifying the full diffusivity tensor to the existing literature. The aim behind the work is to allow for simple extraction of the anisotropic diffusivity tensor from a FRAP test with a circular (or elliptical) ROI in the spatial domain. The approach, which is based on solving the diffusion equation with a Gaussian distribution as the initial condition, is direct, and can be implemented using a commercially available software (Mathematica in our case) by fitting the pixel values of the normalised intensity data with the analytical solution.

To test and to validate the approach, we performed FRAP tests of 3kDa FITC-dextran solution in porcine MCL tissues, and found the anisotropy ratio of the diffusivity tensor, which was close to the results obtained by [11]. We addressed several factors that can impact the diffusivity measurements, such as the initial bleaching profile, the optical slice, the effective diffusivity, the time window for analysis, as well as the radius and intensity of bleaching. We introduced an anisotropic form for the initial Gaussian condition in Equation (4). Since we are using a laser-scanning microscope, the bleaching (and scanning) occurs from the top to the bottom of the bleached ROI. We noticed upon fitting the data of the bleached ROI that its profile may deviate slightly from the preset circle. This deviation would not have a significant impact in the isotropic case, but would affect the anisotropy and, thus, the use of the anisotropic initial condition which minimises this effect. As for the optical slice, we showed with separate experiments performed using a solution of 3kDa FITC-dextran that the planar assumption for diffusion recovery holds for the pinhole opening used. The experimental results for the spherical part of diffusivity, $170 \pm 1.17 \mu \mathrm{m}^{2} / \mathrm{s}$ (SE), were very close to the theoretical value in the Stokes-Einstein equation at $175 \mu \mathrm{m}^{2} / \mathrm{s}$, Equation (38). In these tests, the anisotropy ratio, expected to be exactly 1 in the case of fluid, deviated slightly at $1.04 \pm 0.0016$ (SE). This standard error, besides the standard error of the diffusivity at $1.17 \mu \mathrm{m}^{2} / \mathrm{s}$, could be attributed to the fact that fluid drifting cannot be entirely prevented. Moreover, the anisotropic ratio may also be viewed as a measure of the threshold for the accuracy of the fitting approach.

After spatially fitting the experimental data and obtaining measures of the diffusivity components for each postbleached frame, we suggested using the tensor of inertia for finding an effective measure 
for each diffusivity component. One major advantage of using the moments of inertia is that not only are they affine in the diffusivity components, but they also form a system of independent equations in the diffusivity components. As a consequence, for a given diffusivity component, the fitted values of that component, obtained from the postbleached images, should follow a line once they are substituted in Equation (28). Based on the goodness of fit for this line, one can be confident that the effective diffusivity components are accurate, Figure 7. Although our use of the tensor of inertia in this work is limited to finding effective diffusivity measures, we believe that its simple form, Equation (28), makes it a promising potential measure for diffusion anisotropy that can be used as a stand-alone method.

In Section 5, we addressed the issues related to fitting the data of a bounded domain to the solution of the unbounded domain, and investigated the upper bound for the error. The parameters studied were the size of the bleached region (radius $r$ ) and the intensity of bleaching (parameter $k$ ). For the tested values of $r$ and $k$, and within the time window used for the analysis, the error was small. Additional error analyses were performed for different values of $k$ and $r$ to test the sensitivity of the errors to these parameters, which can be helpful in choosing the radius of the bleached region and the bleaching intensity. It should be noted that such errors will also be present in other approaches of FRAP analysis, as they arise from the nature of FRAP experiments. A limitation of the method in the current form is that it assumes that all molecules are mobile and, therefore, the recovery is complete. This assumption can be corrected for by following a procedure similar to that adopted by [1] (see the unnumbered equation at the bottom of page 1061 in their paper).

In summary, the goal of this work was to introduce a practical approach for extracting the full anisotropic diffusivity tensor from a FRAP test in spatial coordinates, with only a few steps that can be directly implemented with a commercially available software.

\section{Acknowledgements}

The Authors gratefully acknowledge the help of Ruth Seerattan for the help with sample preparation and of Elyar Asl Sabbaghian Hokm Abadi for useful discussions. This work was supported in part by the Natural Sciences and Engineering Research Council of Canada, through the NSERC Discovery Programme [SF, WH], the Canadian Institutes of Health Research (CIHR) [WH], the Canada Research Chair Programme [WH], the Killam Foundation [WH], Dipartimento di Eccellenza 20182022, Politecnico di Torino, Project no. E11G18000350001 [AG, ART], the Biomedical Engineering Graduate Programme of the University of Calgary (Canada), through the BME GP Academic Award $[\mathrm{KH}]$ and the BME Research Scholarship Award [KH], the University of Calgary Eyes High Doctoral Programme $[\mathrm{KH}]$ and the Canadian Society for Biomechanics, through the CSB PhD Student Travel Grant $[\mathrm{KH}]$. Part of this work was conducted during the visit of KH at the Department of Mathematical Sciences (DISMA) "G.L. Lagrange", Politecnico di Torino, under the supervision of AG and with the support of the CSB PhD Student Travel Award.

\section{Competing Interests}

The authors declare that they have no competing interests.

\section{References}

[1] Daniel Axelrod, DE Koppel, J Schlessinger, Ei Elson, and Watt W Webb. Mobility measurement by analysis of fluorescence photobleaching recovery kinetics. Biophysical Journal, 16(9):10551069, 1976.

[2] Haim Brezis. Functional Analysis, Sobolev Spaces and Partial Differential Equations. Springer, 2010 . 
[3] Nancy Burton-Wurster and George Lust. Fibronectin and proteoglycan synthesis in long term cultures of cartilage explants in Ham's F12 supplemented with insulin and calcium: effects of the addition of TGF- $\beta$. Archives of Biochemistry and Biophysics, 283(1):27-33, 1990.

[4] A. Curnier, Q.-C. He, and P. Zysset. Conewise linear elastic materials. Journal of Elasticity, $37: 1-38,1995$.

[5] Chris D DiDomenico, Marianne Lintz, and Lawrence J Bonassar. Molecular transport in articular cartilage - what have we learned from the past 50 years? Nature Reviews Rheumatology, 14(7):393-403, 2018.

[6] Peter Jönsson, Magnus P Jonsson, Jonas O Tegenfeldt, and Fredrik Höök. A method improving the accuracy of fluorescence recovery after photobleaching analysis. Biophysical Journal, 95(11):5334-5348, 2008.

[7] Minchul Kang, Charles A Day, Emmanuele DiBenedetto, and Anne K Kenworthy. A quantitative approach to analyze binding diffusion kinetics by confocal frap. Biophysical Journal, 99(9):27372747,2010 .

[8] Minchul Kang, Charles A Day, Anne K Kenworthy, and Emmanuele DiBenedetto. Simplified equation to extract diffusion coefficients from confocal frap data. Traffic, 13(12):1589-1600, 2012.

[9] Denis Le Bihan, Jean-François Mangin, Cyril Poupon, Chris A Clark, Sabina Pappata, Nicolas Molko, and Hughes Chabriat. Diffusion tensor imaging: concepts and applications. Journal of Magnetic Resonance Imaging, 13(4):534-546, 2001.

[10] Holly A Leddy and Farshid Guilak. Site-specific molecular diffusion in articular cartilage measured using fluorescence recovery after photobleaching. Annals of Biomedical Engineering, 31(7):753$760,2003$.

[11] Holly A Leddy, Mansoor A Haider, and Farshid Guilak. Diffusional anisotropy in collagenous tissues: fluorescence imaging of continuous point photobleaching. Biophysical Journal, 91(1):311316, 2006.

[12] Jeong Ik Lee, Masato Sato, Kiminori Ushida, and Joji Mochida. Measurement of diffusion in articular cartilage using fluorescence correlation spectroscopy. BMC Biotechnology, 11(1):19, 2011.

[13] NF Lori, E Akbudak, JS Shimony, TS Cull, AZ Snyder, RK Guillory, and TE Conturo. Diffusion tensor fiber tracking of human brain connectivity: aquisition methods, reliability analysis and biological results. NMR in Biomedicine, 15(7-8):494-515, 2002.

[14] Alice Maroudas. Physicochemical properties of cartilage in the light of ion exchange theory. Biophysical Journal, 8(5):575-595, 1968.

[15] Hong Qian, Michael P Sheetz, and Elliot L Elson. Single particle tracking. analysis of diffusion and flow in two-dimensional systems. Biophysical Journal, 60(4):910-921, 1991.

[16] Changcheng Shi, Jonathan Kuo, P Darwin Bell, and Hai Yao. Anisotropic solute diffusion tensor in porcine TMJ discs measured by frap with spatial fourier analysis. Annals of Biomedical Engineering, 38(11):3398-3408, 2010.

[17] Brian L Sprague, Robert L Pego, Diana A Stavreva, and James G McNally. Analysis of binding reactions by fluorescence recovery after photobleaching. Biophysical Journal, 86(6):3473-3495, 2004.

[18] Alan S Verkman. Solute and macromolecule diffusion in cellular aqueous compartments. Trends in Biochemical Sciences, 27(1):27-33, 2002. 
[19] Y Xia, T Farquhar, N Burton-Wurster, M Vernier-Singer, G Lust, and LW Jelinski. Self-diffusion monitors degraded cartilage. Archives of Biochemistry and Biophysics, 323(2):323-328, 1995.

[20] Yang Xia, Tony Farquhar, Nancy Burton-Wurster, Edward Ray, and Lynn W Jelinski. Diffusion and relaxation mapping of cartilage-bone plugs and excised disks using microscopic magnetic resonance imaging. Magnetic Resonance in Medicine, 31(3):273-282, 1994. 